\title{
ProNGF $\backslash$ NGF imbalance triggers learning and memory deficits, neurodegeneration and spontaneous epileptic-like discharges in transgenic mice
}

\author{
C Tiveron ${ }^{1}$, L Fasulo ${ }^{1,8}$, S Capsoni ${ }^{2,8}$, F Malerba ${ }^{1,2}$, S Marinelli ${ }^{3}$, F Paoletti ${ }^{1}$, S Piccinin ${ }^{4}$, R Scardigli ${ }^{1,5}$, G Amato ${ }^{1}$, R Brandi ${ }^{1}$, \\ P Capelli $^{2}$, S D’Aguanno ${ }^{6,7}$, F Florenzano ${ }^{1}$, F La Regina ${ }^{1}$, A Lecci ${ }^{1}$, A Manca ${ }^{1}$, G Meli ${ }^{1}$, L Pistillo ${ }^{1}$, N Berretta ${ }^{7}$, R Nistico ${ }^{7}$, F Pavone $^{3}$ \\ and $A$ Cattaneo*,1,2
}

ProNGF, the precursor of mature nerve growth factor (NGF), is the most abundant form of NGF in the brain. ProNGF and mature NGF differ significantly in their receptor interaction properties and in their bioactivity. ProNGF increases markedly in the cortex of Alzheimer's disease (AD) brains and proNGF $\backslash$ NGF imbalance has been postulated to play a role in neurodegeneration. However, a direct proof for a causal link between increased proNGF and AD neurodegeneration is lacking. In order to evaluate the consequences of increased levels of proNGF in the postnatal brain, transgenic mice expressing a furin cleavage-resistant form of proNGF, under the control of the neuron-specific mouse Thy1.2 promoter, were derived and characterized. Different transgenic lines displayed a phenotypic gradient of neurodegenerative severity features. We focused the analysis on the two lines TgproNGF\#3 and TgproNGF\#72, which shared learning and memory impairments in behavioral tests, cholinergic deficit and increased A $\beta$-peptide immunoreactivity. In addition, TgproNGF\#3 mice developed A $\beta$ oligomer immunoreactivity, as well as late diffuse astrocytosis. Both TgproNGF lines also display electrophysiological alterations related to spontaneous epileptic-like events. The results provide direct evidence that alterations in the proNGF/NGF balance in the adult brain can be an upstream driver of neurodegeneration, contributing to a circular loop linking alterations of proNGF/NGF equilibrium to excitatory/inhibitory synaptic imbalance and amyloid precursor protein (APP) dysmetabolism.

Cell Death and Differentiation (2013) 20, 1017-1030; doi:10.1038/cdd.2013.22; published online 29 March 2013

ProNGF, the precursor of nerve growth factor (NGF), ${ }^{1,2}$ is the most abundant NGF form in the brain. ${ }^{3}$ Compared with mature NGF, proNGF is a preferred ligand for $p 75^{\mathrm{NTR}}$ than for TrkA. ${ }^{4}$ ProNGF is cleaved intracellularly to NGF by furin convertase, ${ }^{5}$ and by extracellular proteases (plasmin and metalloproteases). ${ }^{4,6,7}$ ProNGF has functions distinct from those of mature NGF, as mediator of cell death in neurons, oligodendrocytes and cell lines expressing p75 NTR 8,9 The VPS10pfamily receptor sortilin, in association with $p 75^{\mathrm{NTR}}$, is a specific proNGF receptor. ${ }^{10} \mathrm{NGF}$ and proNGF activate distinct transcriptional signatures in target cells, highlighting their different bioactivities. ${ }^{11}$ Therefore, the role initially attributed to the pro-domain, that is, assistance to proper folding, ${ }^{12}$ represents only part of a complex picture. The ratio between expression and signaling of TrkA ${ }^{\text {NTR }}$ and $p 75^{\text {NTR }}$ is critical to determine the prevalence of a trophic or an apoptotic signaling, respectively. ${ }^{13-16}$ Similarly, the balance between proNGF and NGF levels and signaling in the brain is a key determinant for brain homeostasis, with its disruption possibly leading to neurodegeneration. ${ }^{17,18}$ Thus, it was proposed that Alzheimer's disease (AD) neurodegeneration could be linked to unbalanced signaling by proNGF $\backslash$ NGF. Consistently, proNGF accumulates in AD cortex, ${ }^{3,19}$ whereas TrkA expression is reduced and $p 75^{\text {NTR }}$ expression is unaltered. ${ }^{20,21}$ However, it is not known whether accumulation of proNGF is a cause or a consequence of the disease.

To directly examine the consequences of increased proNGF expression, we generated transgenic (Tg) mice expressing furin cleavage-resistant proNGF in early postnatal and adult brain in a background of normal endogenous proNGF $\backslash$ NGF production.

\section{Results}

Production of proNGF transgenic mice expressing furin-resistant proNGF. To study the consequences of an unbalanced proNGF $\backslash$ NGF ratio, we generated transgenic mice expressing furin-resistant mouse proNGF in a

\footnotetext{
${ }^{1}$ Neurotrophic Factors and Neurodegenerative Diseases Unit, EBRI-European Brain Research Institute, Rome, Italy; ${ }^{2}$ Scuola Normale Superiore, Pisa, Italy; ${ }^{3} \mathrm{CNR}$, Cell Biology and Neurobiology Institute (IBCN), Roma, Italy; ${ }^{4}$ Pharmacology of Synaptic Plasticity Unit, EBRI-European Brain Research Institute, Rome, Italy; ${ }^{5} \mathrm{CNR}-\mathrm{IFT}$, Roma, Italy; ${ }^{6}$ University of Rome Tor Vergata, Roma, Italy and ${ }^{7}$ Fondazione Santa Lucia IRCCS, Roma, Italy

${ }^{*}$ Corresponding author: A Cattaneo, Scuola Normale Superiore, Piazza dei Cavalieri 7, 56126 Pisa, Italy. Tel: + 3950509320 ; Fax: + 39503153210 ; E-mail: antonino.cattaneo@sns.it

${ }^{8}$ Joint first authors.

Keywords: neurotrophin; Alzheimer; cholinergic deficit; NGF; proNGF; neurodegeneration

Abbreviations: NGF, nerve growth factor; AD, Alzheimer's disease; A $\beta$, amyloid- $\beta$; APP, amyloid precursor protein; IRES, internal ribosome entry site sequence; EGFP, green fluorescent protein; CTX, cortex; WT, wild type; AA, active avoidance test; ORT, object recognition test; MWM, Morris water maze test; ChAT, choline acetyltransferase; BFCN, basal forebrain cholinergic neurons; HP, hippocampus; BF, basal forebrain; GFAP, glial fibrillar acidic protein; ScFv, single-chain antibody fragment; $\mathrm{A} \beta \mathrm{O}, \mathrm{A} \beta$ oligomers; $\mathrm{EC}$, entorhinal cortex; LTP, long-term potentiation; IP, immunoprecipitation; Tg, transgenic; DS, Down syndrome; $\mathrm{E} \backslash$, excitatory $\backslash$ inhibitory; $\mathrm{MCl}$, mild cognitive impairment; MMP, matrix metalloproteinase; $\mathrm{DG}$, dentate gyrus

Received 27.7.12; revised 14.2.13; accepted 18.2.13; Edited by L Greene; published online 29.3.13
} 
background of normal endogenous proNGF $\backslash$ NGF production. The proNGF cleavage site for furin (RSKR) was mutated to RSAA. $^{8}$

The proNGF expression cassette (Figure 1a) contains the promoter of mouse Thy 1.2 gene. ${ }^{22}$ Expression from this promoter, in the brain of transgenic mice, begins from approximately postnatal days $\mathrm{P} 6-10$ and continues into adulthood, leading to a mosaic regional and cellular distribution, varying among different founder transgenic lines. ${ }^{23,24} \mathrm{An}$ internal ribosome entry site sequence (IRES) ensures transcription of a bicistronic mRNA, driving coexpression of pre-proNGF with the green fluorescent protein (EGFP) reporter (Figure 1a). Four founder lines expressing the transgene were identified: TgproNGF\#3, \#17, \#36 and \#72 (transgene copy number/mouse haploid genome are in Supplementary Table S1).

Mice from the different lines had normal brain size and overall structure, with the exception of mice TgproNGF\#17, which showed hydrocephalus and were excluded from subsequent analyses.

Homozygous mice could be successfully derived only for TgproNGF\#72, whereas the other founders were bred as heterozygous lines. TgproNGF\#36 had reduced fertility, which limited the availability of mice from this line.

Transgene expression was evaluated by EGFP immunohistochemistry (Figure 1b), as anti-proNGF antibodies do not discriminate endogenous proNGF from transgene-encoded proNGF (Figure 1c). All transgenic mice displayed EGFP expression in the cortex (CTX) and hippocampus (HP; Figures $1 \mathrm{~b}-\mathrm{d}$ ) at varying expression levels (Supplementary Table S2). Double immunofluorescence showed that EGFP-positive cells also express significant levels of proNGF (Figure 1d). mRNA analysis confirmed overexpression of transgene with respect to endogenous NGF mRNA (see below).

The proNGF content in both transgenic lines was several fold higher than in wild type (WT; Figure 1e). The biochemical analysis is described below.

\section{Behavioral phenotype of TgproNGF mice: learning} and or memory deficit. We assessed learning and memory deficits in P90 TgproNGF\#3, \#72 and \#36 mice by means of the active avoidance (AA), object recognition (ORT) and Morris water maze (MWM) tests.

In the AA test (Figure 2a), TgproNGF\#3 mice showed almost no conditioned responses in all training sessions, revealing a dramatic impairment in avoidance acquisition, a deficit also found in TgproNGF\#36 mice, although less pronounced. On the other hand, the avoidance responses of TgproNGF\#72 mice did not differ from those of WT mice. Poor avoidance learning in TgproNGF\#3 transgenic line is not due to reduced locomotor activity (Figure $2 b$ ).

Because of limited availability of TgproNGF\#36 mice, subsequent behavioral analyses focusing on the characterization of working and spatial memory deficits were carried out in heterozygous TgproNGF\#3 and in homozygous Tgpro NGF\#72 mice.

In the habituation phase of the ORT, TgproNGF\#3 showed complete immobility in the entrance corner of the maze (Figure 2c). In the sample phase, TgproNGF\#3 explored the two identical objects for a much shorter time than WT mice
(Figure 2d). Because of these behavioral responses, suggesting increased anxiety, TgproNGF\#3 were not analyzed in the subsequent test phase of ORT. TgproNGF\#72 mice showed normal exploratory behavior in the sample phase (Figure 2d), whereas, in the test phase (Figure 2e) they spent equal time exploring the new and the familiar object, unlike WT mice, which explored more the new object. Thus, TgproNGF\#72 mice show working memory deficit.

Spatial learning and memory were assessed by MWM test. The swimming speed of TgproNGF\#3, TgproNGF\#72 and WT mice was identical (Figure 2f). In the acquisition phase, TgproNGF\#72 mice showed a similar degree of learning than WT mice (Figure 2g), whereas learning in TgproNGF\#3 mice was dramatically impaired (Figure $2 \mathrm{~g}$ ). In the retention phase, whereas WT and proNGF\#72 mice remembered the target quadrant (Figure 2h), TgproNGF\#3 mice were unable to remember where the platform was located. Thus, P90 TgproNGF\#3 mice showed a severe learning and memory deficit.

In conclusion, the behavioral tests showed significant learning and memory impairments in different transgenic lines, more pronounced in TgproNGF\#3 mice.

Cholinergic deficit and neurodegenerative traits. In order to investigate the possible mechanisms underlying memory deficits in TgproNGF mice, the basal forebrain cholinergic (BFC) system was analyzed in P90 mice. Choline acetyltransferase (ChAT) immunohistochemistry (Figure 3a) revealed a severe deficit in TgproNGF\#3 mice: the number of ChAT-immunoreactive neurons in the medial septum was reduced by $40 \%$ (Figure $3 \mathrm{~b}$ ). The plexus of septal cholinergic fibers also appeared greatly reduced, and basal forebrain cholinergic neuron (BFCN) atrophy was observed (Figure 3c).

In P90 TgproNGF\#72 mice, ChAT-positive BFCNs in the septum were not reduced or atrophic (Figure $3 b$ ). However, TgproNGF\#72 mice showed decreased cholinergic innervation in the CTX (Figure 3d, quantification in Supplementary Figure S1A).

We conclude that TgproNGF\#3 mice have a cholinergic deficit more severe than TgproNGF\#72 mice, consistent with the more pronounced behavioral phenotype. Interestingly, no cholinergic deficit is observed in TgproNGF\#3 mice at P15 and P30, when the transgene product just starts to be produced and to accumulate (Supplementary Figures S1B and $\mathrm{C}$ and S6).

Links between imbalance in proNGF/NGF signaling in the brain and activation of amyloid precursor protein (APP) processing have been recently postulated. ${ }^{17,18} \mathrm{We}$ therefore evaluated changes in the expression of APP-derived amyloid$\beta$ ( $\mathrm{A} \beta$ ) peptide. The $\mathrm{A} \beta$ immunoreactivity was visualized with $\mathrm{mAb} 4 \mathrm{G} 8$, specific for $A \beta$ residues (17-24). In P90 TgproNGF\#72 mice, a specific intracellular $A \beta$ staining was observed in layer II of the entorhinal CTX with a fourfold increase in the number of $\mathrm{A} \beta$-positive neurons (Figures $3 \mathrm{e}$ and $\mathrm{g}$ ). A specific anti-A $\beta$ amyloid immunostaining was also observed in neurons expressing proNGF in other regions, such as hippocampal dentate gyrus (DG) and CA1 (not shown). In TgproNGF\#3 mice, a few neurons displayed increased mAb 4G8 immunoreactivity in the CTX (Figure 3f), whereas at 12 months of age, $\mathrm{A} \beta$ immunoreactivity was 
a

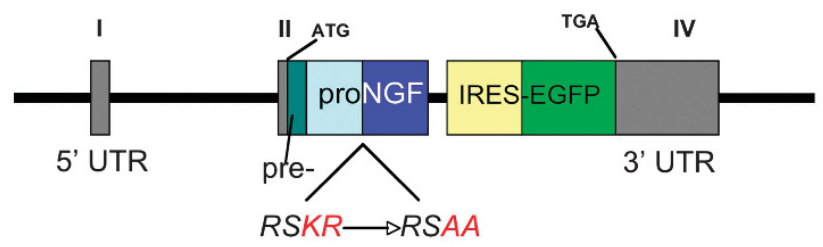

(mutation in furin cleavage site)

b

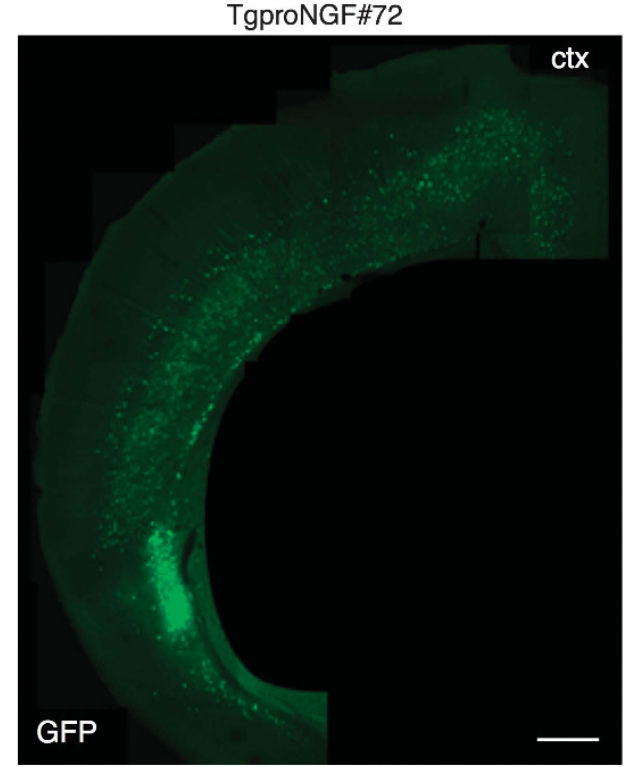

。

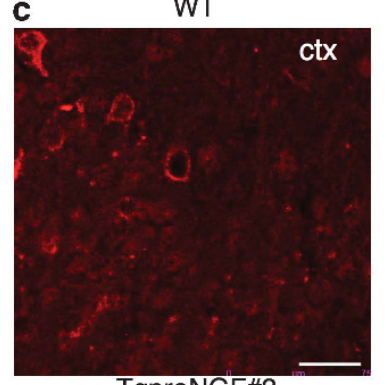

TgproNGF\#3

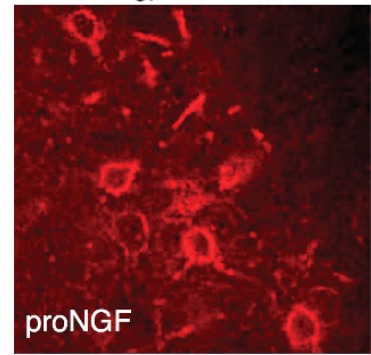

d

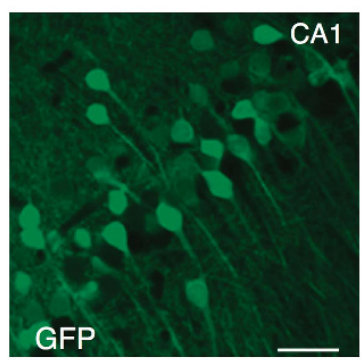

TgproNGF\#72
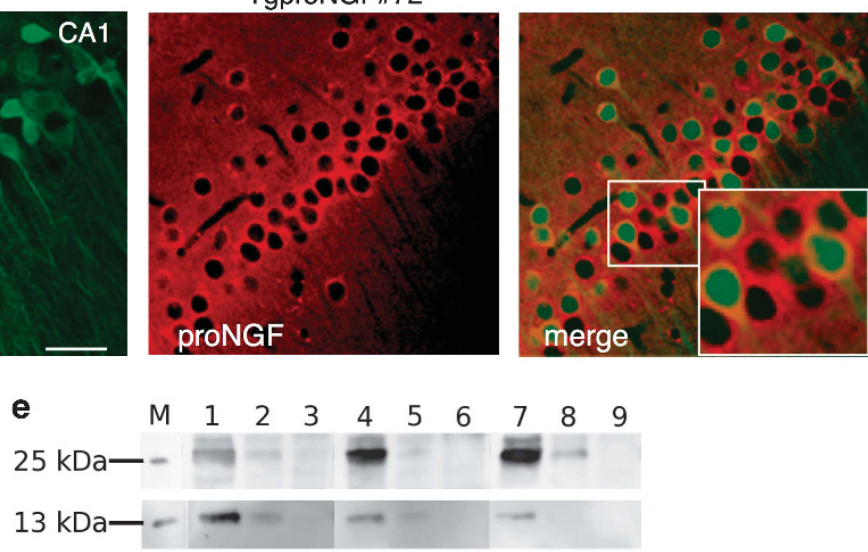

Figure 1 Production of the TgproNGF mice. (a) Schematic representation of the Thy 1.2 expression cassette. The Thy 1.2 vector contains $6.5 \mathrm{~kb}$ of the murine thy 1.2 gene, encompassing the regulatory sequences driving the selective expression in the adult brain (exons I, II and IV responsible for expression in neurons, shown in gray). The internal ribosome entry site (IRES) of the encephalomyocarditis (ECMV) virus enables the transcription of a bicistronic mRNA, coexpressing the mutated pre-pro-NGF and the enhanced green fluorescent protein (EGFP) as a reporter. Scheme of NGF transcript: NGF is synthesized as pre-proNGF (the pre-sequence is the 18 amino acid (aa) long secretory leader sequence); the pro-sequence is cleaved at specific dibasic aa sites by furin or by other proteases. In the furin-resistant mutant, the pre-proNGF cDNA (pre: leader sequence; pro-sequence; mature NGF) is mutagenized at the dibasic aa included in the furin-recognition site RSKR to RSAA (corresponding to the sequences -4 to -1 of proNGF protein). (b-d) EGFP and proNGF expression in the cortex and hippocampus of TgproNGF mice. (b) EGFP expression in the cortex of TgproNGF\#72 mice. (c) Anti-proNGF immunofluorescence (in red): endogenous proNGF expression in WT mice (splenial cortex). In Tg mice (TgproNGF\#3 mice), proNGF-positive neurons are more numerous and more immunoreactive than in WT mice. Anti-proNGF staining does not discriminate between endogenous proNGF or proNGF encoded by the transgene (rabbit polyclonal antibody). (d) In the hippocampus, high expression levels of EGFP are found in the CA1 region. EGFP reporter expression identifies neurons coexpressing transgenic proNGF (mRNA transcript is bicistronic). Double labeling shows coexpression of EGFP (green) and proNGF (in red, staining with scFv-anti-proNGF recombinant antibody in the format of single-chain Fv fragment (scFv)). Inset $\times 2.5$. Scale bar: (b) $750 \mu \mathrm{m}$, (c) $25 \mu \mathrm{m}$, (d) $80 \mu \mathrm{m}$. (e) Immunoprecipitation on extracts from various brain areas (hippocampus (HP), basal forebrain (BF) and cortex (CTX)) with anti-NGF $\alpha$ D11 antibody, followed by western blot (WB) with anti-NGF or anti-proNGF antibody, as described in Materials and Methods. A representative WB probed with anti-proNGF (top) or anti-NGF (bottom) is shown. M, recombinant protein marker (proNGF top, NGF bottom); 1: IP on \#72 male mouse, HP, 3 months; 2: IP on \#3 male mouse, HP, 3 months; 3 : IP on WT male mouse, HP, 3 months; 4 : IP on \#72 male mouse, BF, 3 months; 5: IP on \#3 male mouse, BF, 3 months; 6 : IP on WT male mouse, BF, 3 months; 7: IP on \#72 male mouse, CTX, 3 months; 8: IP on \#3 male mouse, CTX, 3 months: 9 : IP on WT male mouse, CTX, 3 months 

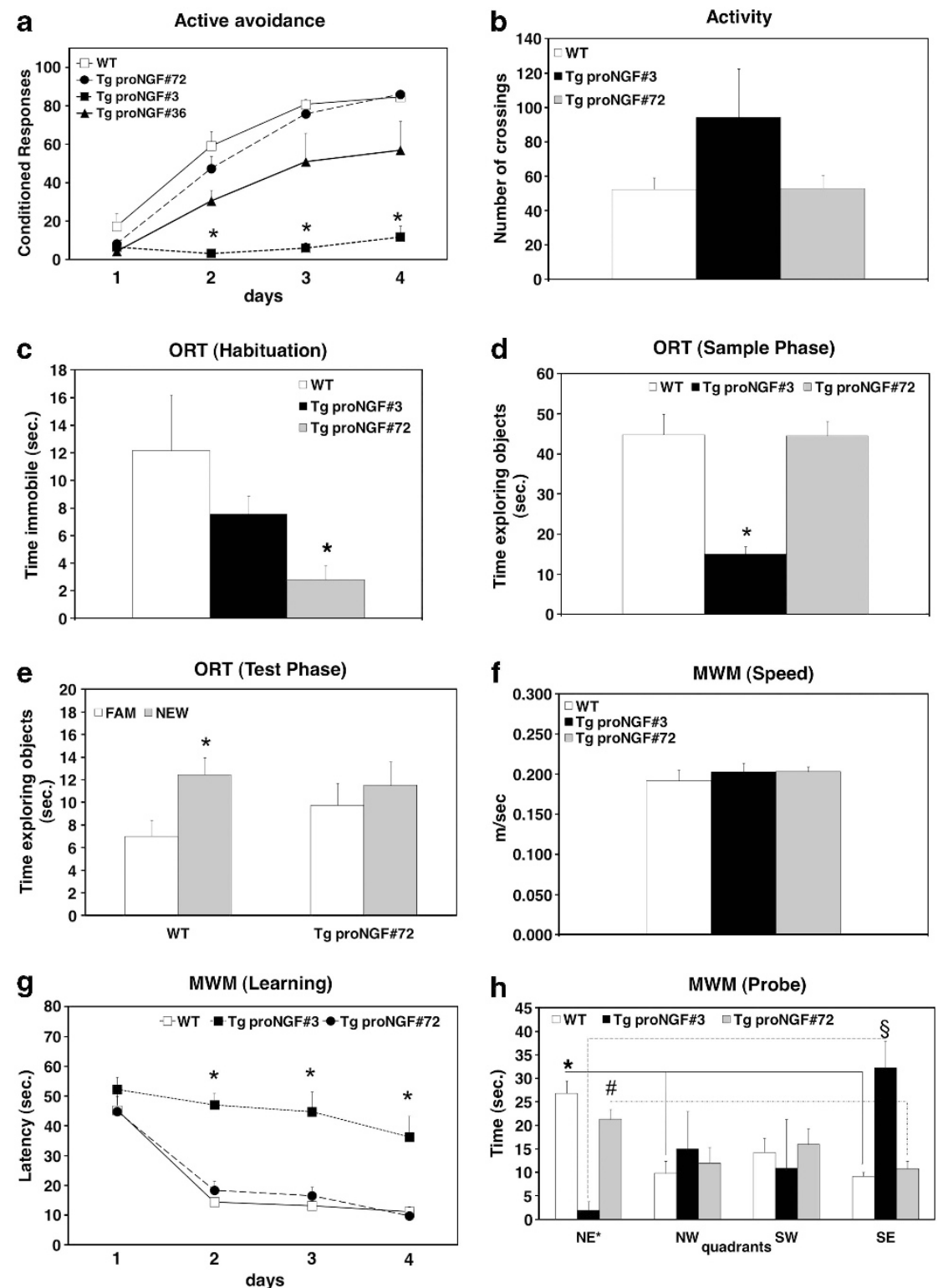

Figure 2 Behavioral analysis shows impairment in learning and memory in P90 TgproNGF mice. (a) Active avoidance. (b) Activity. Object recognition test: (c) habituation, (d) sample phase and (e) test phase. Morris water maze test: (f) swimming velocity, (g) learning and (h) memory test. Bars and points are representative of mean \pm S.E.M. In $(\mathbf{a}, \mathbf{c}, \mathbf{d}$ and $\mathbf{g}){ }^{*} P<0.05$ versus WT mice. In $(\mathbf{e}){ }^{*} P<0.05$ new versus familiar object. In (h) ${ }^{*}$ and solid line indicate $P<0.05$ target quadrants versus adjacent and opposite quadrants in WT mice. " and broken/dotted line indicate $P<0.05$ target quadrants versus adjacent and opposite quadrants in TgproNGF\#72. $\S$ and broken line $=P<0.05$ in TgproNGF\#3 mice. (h) In the retention phase, whereas WT (Kruskal-Wallis test and post hoc Bonferroni test: NE* versus NW $P=0.003 ; \mathrm{NE}^{*}$ versus SW $P=0.06$; NE* versus SE $P=0.002$ ) and proNGF\#72 mice (Kruskal-Wallis test and post hoc Bonferroni test: NE* versus NW $P=0.06$; NE* versus $S W P=0.42 ; \mathrm{NE}^{*}$ versus SE $P=0.03$ ) remembered the target quadrant, TgproNGF\#3 mice were unable to remember where the platform was located, exploring more the SE adjacent quadrant (Kruskal-Wallis test and post hoc Bonferroni test: $\mathrm{NE}^{*}$ versus NW $P=0.63 ; \mathrm{NE}^{*}$ versus SW $P=0.96 ; \mathrm{NE}^{*}$ versus $\mathrm{SE} P=0.03$ )

severely enhanced (see below). The $\mathrm{A} \beta$ immunoreactivity was also observed with other anti-A $\beta$ amyloid antibodies ( $\mathrm{N}$-terminal or C-terminal antiamyloid antibodies in TgproNGF\#72 and \#3 mice, respectively, data not shown).

TgproNGF mice showed no reduction of $\operatorname{trkA}(+)$ and p75(+) neurons in the CTX, HP and basal forebrain (BF) (Supplementary Figures S2A and B and S4).

Among the cells that express TrkA and p $75^{\mathrm{NTR}}$ receptors, astrocytes represent a major cellular target, where the consequences of a proNGF/NGF imbalance could be exerted.
The phenotype of astrocytes was therefore evaluated by glial fibrillar acidic protein (GFAP) immunofluorescence. At P90, TgproNGF\#3 mice showed a number of astrocytes in the HP reduced, with respect to WT mice (Figure 4a), in the DG by $\sim 50 \%$ (Figure $4 \mathrm{~b}$ ). At P90, in TgproNGF\#72 mice the astrocyte number was unchanged, compared with WT mice (Figures 4a and b), but they appeared hypertrophic with thicker ramifications. Thus, proNGF/NGF imbalance results in alteration of morphology and/or number of astrocytes at P90. The number of NeuN-positive neurons was unchanged (not shown). 

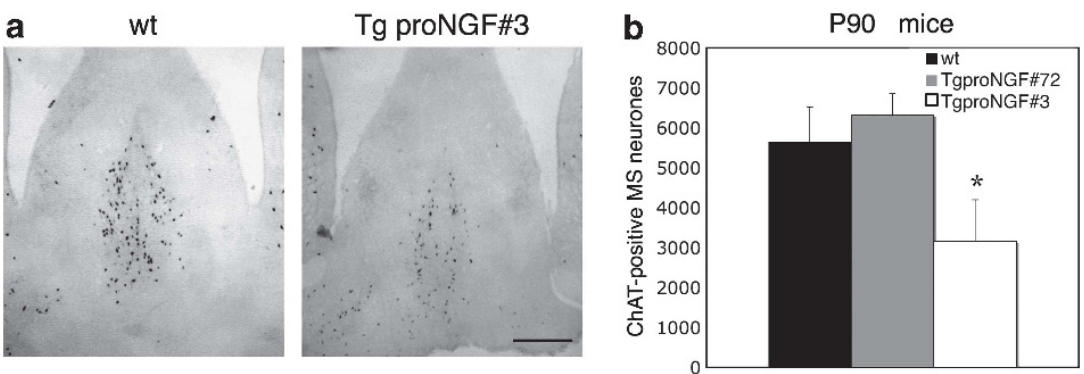

c
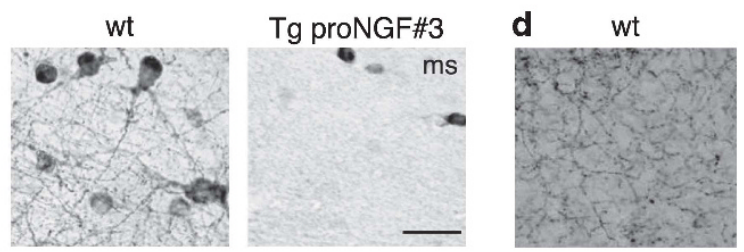

Tg proNGF\#72
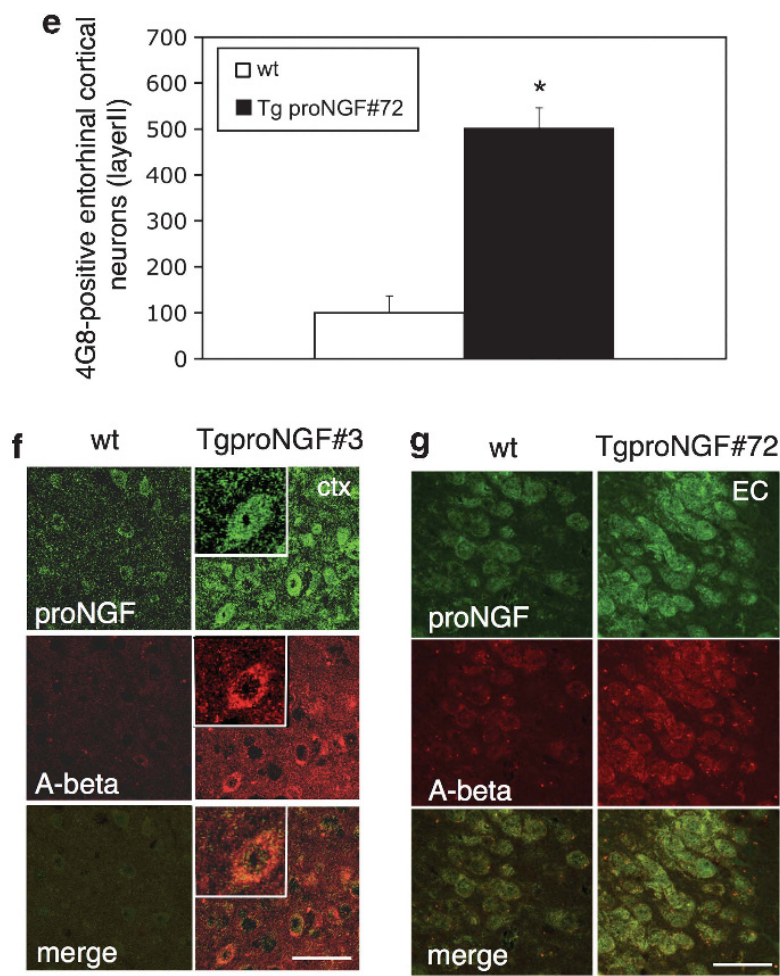

Figure 3 (a-g) Cholinergic deficit and A $\beta$ (4G8 mAb) immunoreactivity in P90 TgproNGF\#3 and proNGF\#72 mice. (a) ChAT immunohistochemistry in P90 TgproNGF\#3 mice: the number of ChAT-immunoreactive neurons in the medial septum is decreased in TgproNGF\#3 mice as compared with WT mice (no reduction is observed in the horizontal or vertical diagonal band of Broca). In TgproNGF\#72 mice, no reduction in ChAT + neurons is observed in the septum. Quantification is shown in (b). Bars and lines are representative of mean \pm S.E.M. ( ${ }^{\star} P<0.05$ versus WT). BFCNs in the medial septum were counted; counting was expressed as stereological counting (at least $3-5$ sections per animal, $n=3-7$ per each animal group). In TgproNGF\#3 mice, cholinergic fibers are also greatly reduced in the septum (c). Scale bar: (a) $40 \mu \mathrm{m}$; (c) $80 \mu \mathrm{m}$. (d) In TgproNGF\#72 mice, although the number of cholinergic neurons in the septum is unaffected, the cholinergic innervation in the cortex is significantly reduced, with a decreased number of cholinergic varicosities. Scale bar: $80 \mu \mathrm{m}$. Quantification is shown in Supplementary Figure S1A. (e) Counting of 4G8-positive neurons in the layer II of the entorhinal cortex of TgproNGF\#72 and WT mice: MAb 4G8 strongly labels the superficial layers of the entorhinal cortex in P90 TgproNGF\#72 mice compared with wild-type mice $(P<0.05)$. Cell counting was performed for each region on three squares of $150 \times 150 \mu \mathrm{m}$ per brain section, at least 3-5 sections per animal $(n=3-7$ per each animal group). (f and g) A $\beta$ immunoreactivity in P90 TgproNGF mice. Confocal microscopy of double immunofluorescence for A $\beta$ (mAb 4G8) and proNGF in TgproNGF mice. Brain sections from P90 WT, TgproNGF\#3 (f) and TgproNGF\#72 (g) mice were labeled with 4G8 and anti-proNGF antibodies. 4G8 immunofluorescence signal is detected in the cytoplasm of a few proNGF-positive neurons in TgproNGF\#3 mice, inset $\times 1.5 \mathrm{zoom}$ (f, splenial cortex). (g) A $\beta$ immunoreactivity in the entorhinal cortex (EC) of TgproNGF\#72 mice colocalizes with proNGF expression. (f and $\mathbf{g}$ ) Scale bar: $25 \mu \mathrm{m}$

Phenotype of aged TgproNGF\#3 mice. The analysis of TgproNGF\#3 mice, showing the most severe phenotype among different lines, was extended to aged animals (12 months old).
Severe deficit in the AA test (Figure 5a) as well as the absence of changes in locomotor activity (Figure $5 b$ ) observed in P90 mice was confirmed in aged animals. 


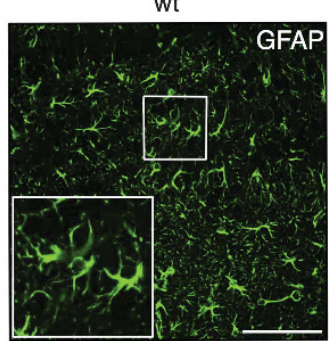

Tg proNGF\#3(P90)

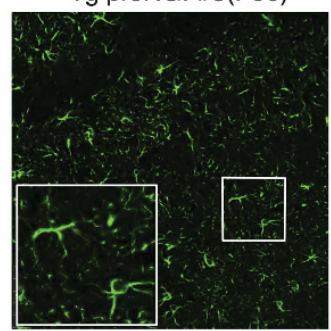

Tg proNGF\#72(P90)

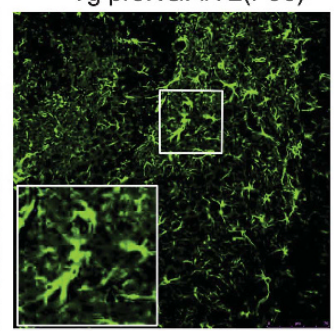

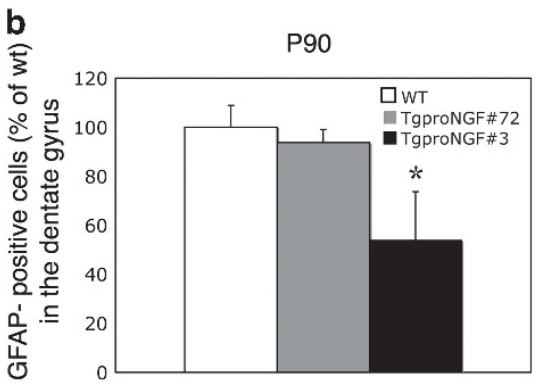

C

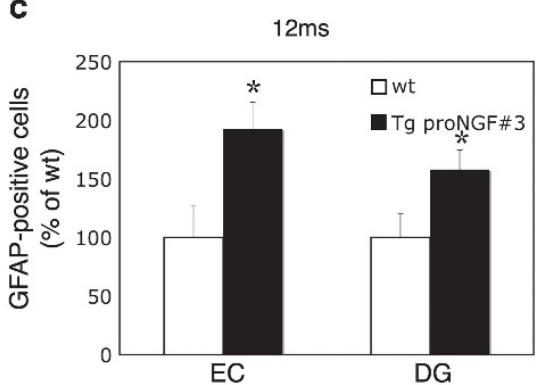

d
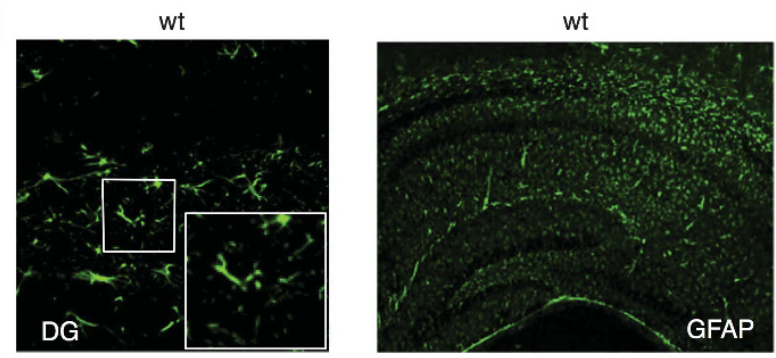

Tg proNGF\#3(12ms)

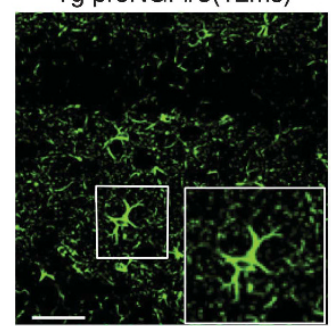

Tg proNGF\#3(12ms)

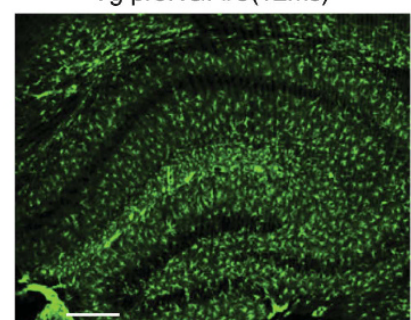

Figure 4 GFAP immunoreactivity in 3-month-old TgproNGF\#3 and proNGF\#72 mice. (a) Glial fibrillar acidic protein (GFAP) immunofluorescence in the dentate gyrus of P90 TgproNGF mice: in TgproNGF\#3 mice, astrocytes are decreased compared with WT mice. Inset $\times 2.5$. In TgproNGF\#72 mice the number of astrocytes is unchanged; however, astrocyte branches appeared thicker. (b) Quantification of astrocytes in the hilar region of the dentate gyrus of the hippocampus in P90 TgproNGF mice ( ${ }^{\star} P<0.001$ versus WT). Bars and lines are representative of mean \pm S.E.M. (a) Scale bar: $100 \mu \mathrm{m}$. Cell counting was performed for each region on three squares of $150 \times 150 \mu \mathrm{m}$ per brain section, at least 3-5 sections per animal ( $n=3-7$ per each animal group). (c and d) GFAP immunoreactivity reveals astrocytosis in the hippocampus of 12-month-old TgproNGF\#3 mice. (c) Increase of GFAP-positive cells in the cortex (entorhinal cortex (EC)) and dentate hilar region (DG) of TgproNGF\#3 mice hippocampus ( $<<0.05$ ). (d) High (hilus of the dentate gyrus) and low magnification of the hippocampus of 12-month-old TgproNGF\#3 and WT mice. Note the increase in GFAP immunoreactivity in the TgproNGF\#3 mice. Inset $\times 2$. (c) Bars and lines are representative of mean \pm S.E.M. (d) Scale bar: $100 \mu \mathrm{m}$ (left panels) and $300 \mu \mathrm{m}$ (right panels). Cell counting was performed for each region on three squares of $150 \times 150 \mu \mathrm{m}$ per brain section, at least $3-5$ sections per animal ( $n=3-7$ per each animal group)

The freezing behavior in object and arena exploration during the habituation phase of ORT was still present (Figure 5c) and the test could not be taken to the end. In the MWM test, the 12-month-old TgproNGF\#3 displayed severe learning (Figure 5e) and memory deficits (Figure 5f), as observed at P90. These mice swam faster than aged-matched WT mice (Figure 5d), which, similar to the freezing behavior in the habituation phase of ORT, suggests a high anxiety behavior.
Histological analysis of the BF nuclei demonstrated a marked reduction in BFCNs in the medial septum and horizontal diagonal band of Broca (Figures 6a and b); the reduction was greater than that at $\mathrm{P} 90$. The average area of residual ChAT-immunoreactive neurones was reduced $(77.6 \pm 22.3 \%)$, as well as the density of septal cholinergic fibers (Figure 6c). BFCN atrophy was not accompanied by decreased number of TrkA and $\operatorname{p75} 5^{\mathrm{NTR}}(+)$ neurons (see below). 
a
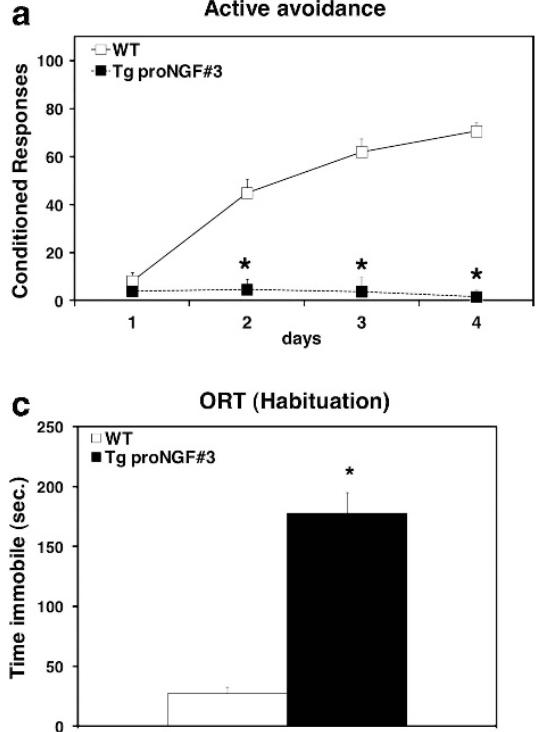

e

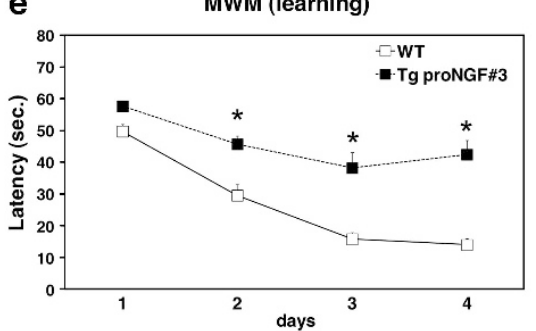

b

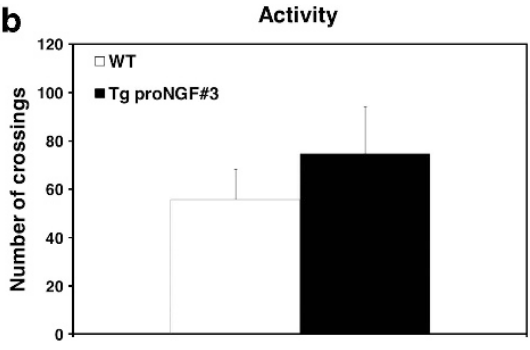

d

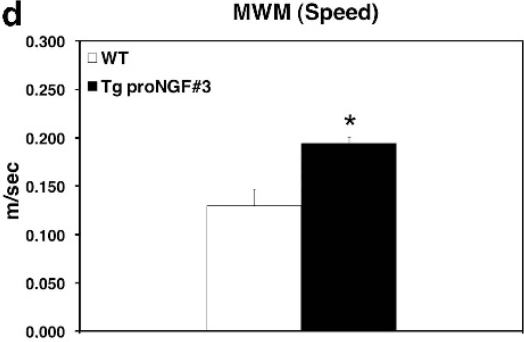

f

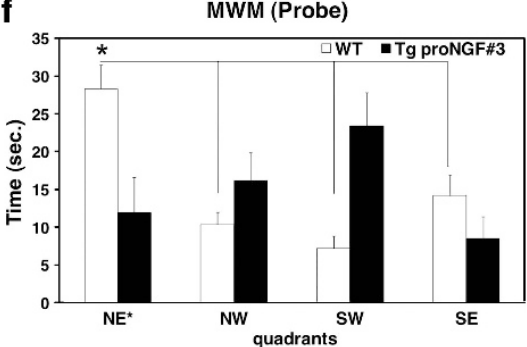

Figure 5 Learning and memory impairment in 12-month-old TgproNGF\#3 mice. (a) Active avoidance. (b) Activity. Object recognition test: (c) habituation. Morris water maze test: (d) swimming velocity, (e) learning and (f) memory test. Bars and points are representative of mean \pm S.E.M. In (c and e) ${ }^{*} P<0.05$ versus WT mice. In (f) ${ }^{*}$ and solid line indicate $P<0.05$ target quadrants versus adjacent and opposite quadrants in WT mice. The 12-month-old TgproNGF\#3 display severe learning (e) and memory deficits (f). During the probe phase of MWM, TgproNGF\#3 explored more, although not in a statistically significant manner, the SW quadrant (Kruskal-Wallis test plus Bonferroni post hoc test $P=0.09$ )

In the 12-month-old TgproNGF\#3 mice, diffuse and vascular (Figure $6 \mathrm{~d}$ ) staining for $\mathrm{A} \beta$ peptide was observed in the CTX and HP, along with a high number of cells showing high intraneuronal staining. Confocal microscopy study showed that the increase in intracellular $\mathrm{A} \beta$ was, very often, localized to proNGF-positive neurons, suggesting a direct link between proNGF expression and intracellular $\mathrm{A} \beta$ production (Figure $6 e$ ). The $A \beta$-oligomeric species are considered the early and more toxic species formed as a consequence of aberrant APP processing. ${ }^{25}$ The presence of $\mathrm{A} \beta$-oligomeric species in TgproNGF\#3 mice was investigated, exploiting ScFvA13, a recombinant single-chain antibody fragment specifically recognizing $A \beta(1-42)$ oligomers $(A \beta O)$, with high selectivity and specificity for $\mathrm{A} \beta$ monomers or fibrillar forms. ${ }^{26}$ In the brains of APP $\backslash$ PS1 (ref. 27) and AD11 (ref. 28) transgenic mice (used as positive controls for human and mouse $\mathrm{A} \beta$ oligomers, respectively), ScFvA13 (anti-A $\beta O$ ) detects clusters of $\mathrm{A} \beta \mathrm{O}$ immunoreactivity in the CA1 hippocampal region (Figure 6f). In TgproNGF\#3 mice, the anti-A $\beta \mathrm{O}$ specifically stains the HP (mainly $\mathrm{CA} 1$ ) and the EC (particularly layer II) (Figure 6f). The $\mathrm{A} \beta \mathrm{O}$ immunoreactivity is absent in WT mice (Figure 6f), or if the anti-tag antibody is omitted (Supplementary Figure S3A).
Tau-related neuropathology, linked to its abnormal phosphorylation pattern, accompanies $\mathrm{A} \beta$-related neurodegeneration in $A D$. No evidence for changes in phospho-tau immunoreactivity was detected in the brain of 12-month-old TgproNGF\#3 mice by Anti-phospho-tau (mAbAT8) immunohistochemistry (Supplementary Figure S3B).

The phenotype of glial cells in 12-month-old TgproNGF\#3 mice was studied by GFAP immunofluorescence, revealing both a higher number of astrocytes and a more ramified morphology (Figure 4d), particularly in cortical and hippocampal regions (quantification in Figure 4c). This is opposite to the reduction observed at P90 (Figures $4 a$ and b), showing that ages of 3 and 12 months correspond to different stages of the neurodegeneration induced by proNGF imbalance in these mice.

Finally, we examined the overall expression of TrkA, p75 ${ }^{\mathrm{NTR}}$ and sortilin receptors in TgproNGF\#3 mice. Western blot analysis from three brain areas (BF, HP and CTX) showed that the levels of $\mathrm{p} 75^{\mathrm{NTR}}$ and sortilin receptors in TgproNGF\#3 were not significantly different from WT mice (Supplementary Figure S2C). Immunofluorescence revealed an overall normal pattern of trkA and p75 expression (Supplementary Figure S4). 
a

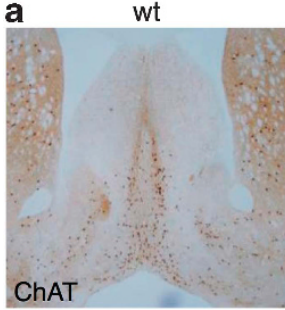

c

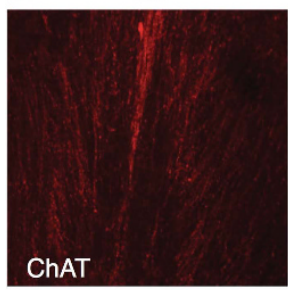

e
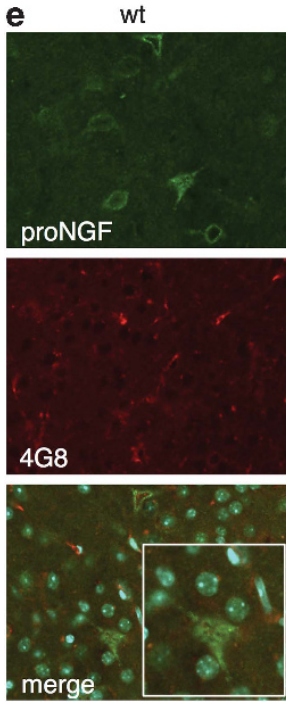

Tg proNGF\#3

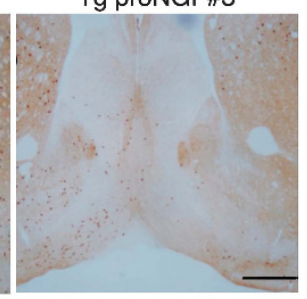

Tg proNGF\#3

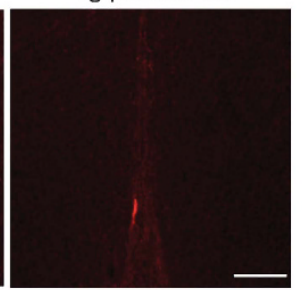

Tg proNGF\#3
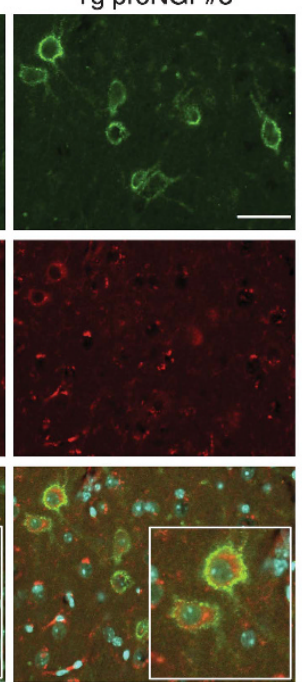

b

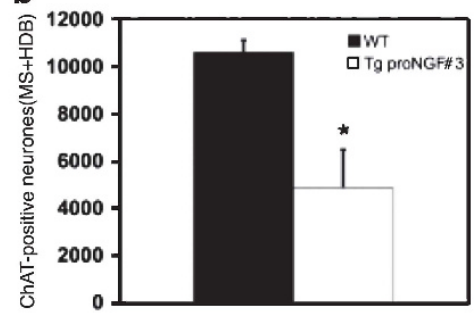

d wt

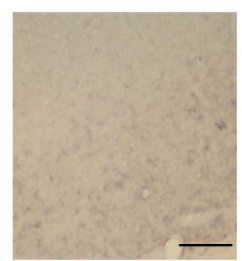
Tg proNGF\#3

f
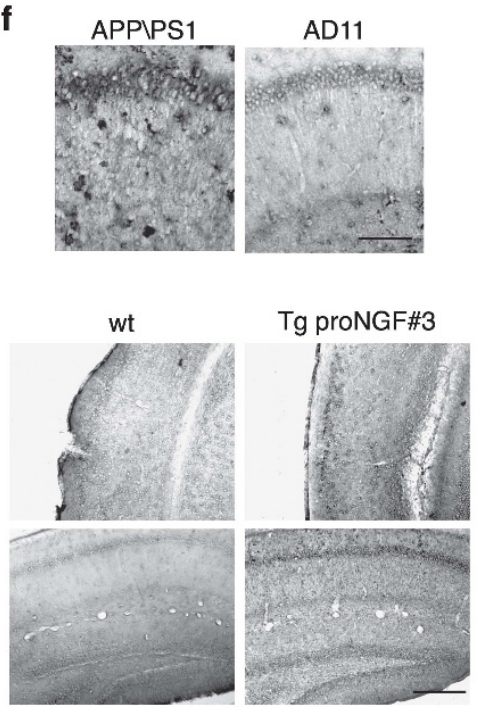

Figure 6 Cholinergic deficit and A $\beta$ immunoreactivity in 12-month-old TgproNGF\#3 mice. (a) ChAT immunohistochemistry in the septal region of 12-month-old TgproNGF\#3 mice: ChAT-immunoreactive neurons are decreased in TgproNGF\#3 mice compared with WT mice. Scale bar: $400 \mu \mathrm{m}$. (b) Quantification of ChATimmunoreactive neurons in the medial septum and horizontal diagonal band $\left({ }^{*} P<0.05\right.$ versus WT). Bars and lines are representative of mean $\pm S$.E.M. BFCNs in the medial septum were counted and counting was expressed as stereological counting. (c) ChAT immunofluorescence: septal cholinergic fibers (in red) also appear greatly reduced. Scale bar: $50 \mu \mathrm{m}$. (d) In the cortex, marked diffuse A $\beta$ immunoreactivity (mAb 4G8) was detected in 12-month-old TgproNGF\#3 mice with respect to WT mice. The arrow points to an immunoreactive vessel. Scale bar: $100 \mu \mathrm{m}$ (d). (e) Brain sections from 12-month-old WT and TgproNGF\#3 mice were labeled for A $\beta$ (mAb 4G8; red channel) and anti-proNGF (green channel), nuclei, blue channel. The A $\beta$ immunofluorescence signal is higher in the cytoplasm of proNGF-positive neurons, although it is also detectable in some proNGF-negative neurons (splenial cortex). Inset $\times 1$.6. Scale bar: $25 \mu \mathrm{m}$. (f) scFvA13 recombinant antibody (in the format of single-chain Fv fragment (ScFv)) specific for oligomeric amyloid species ${ }^{26}$ markedly stains the CA1 region of the hippocampus of 1-year-old APP $\times$ PS1 transgenic mice (with A $\beta$ oligomeric (A $\beta O$ )-immunoreactive clusters) and anti-NGF AD11 mice. The bound ScFv antibody is detected through its Sv5 tag, with an anti-tag antibody. Scale bar: $100 \mu$ m. In 12-month-old TgproNGF\#3 mice, scFvA13 markedly decorates both the entorhinal cortex and the hippocampus in comparison with WT brain. A $\beta O$ immunoreactivity is not seen in sections if the anti-tag antibody is omitted (Supplementary Figure S3A). Scale bar: $250 \mu \mathrm{m}$

Hippocampal synaptic function and spontaneous epileptic-like events in TgproNGF mice. To verify whether the memory deficits observed in TgproNGF\#3 mice correlate with defects in synaptic plasticity, or disequilibrium of the hippocampal synaptic networks, an electrophysiological study was performed. At hippocampal CA1 synapses, no significant differences in stimulus-response curves (Figure 7a) and in paired-pulse facilitation (Figure 7b) were observed between TgproNGF\#3 and control mice, indicating that neither basal transmission nor short-term plasticity was affected in this region. Next, long-term potentiation (LTP) induced by high-frequency stimulation was studied. The magnitude of potentiation measured $50-60$ min after the conditioning train was not significantly different in TgproNGF\#3 (147\% $\pm 8 \%)$ and WT mice (162 $\pm 10 \%$; $P>0.05$; Figure7c).

A common denominator at the electrophysiological level between mouse neurodegeneration models of different kinds, 

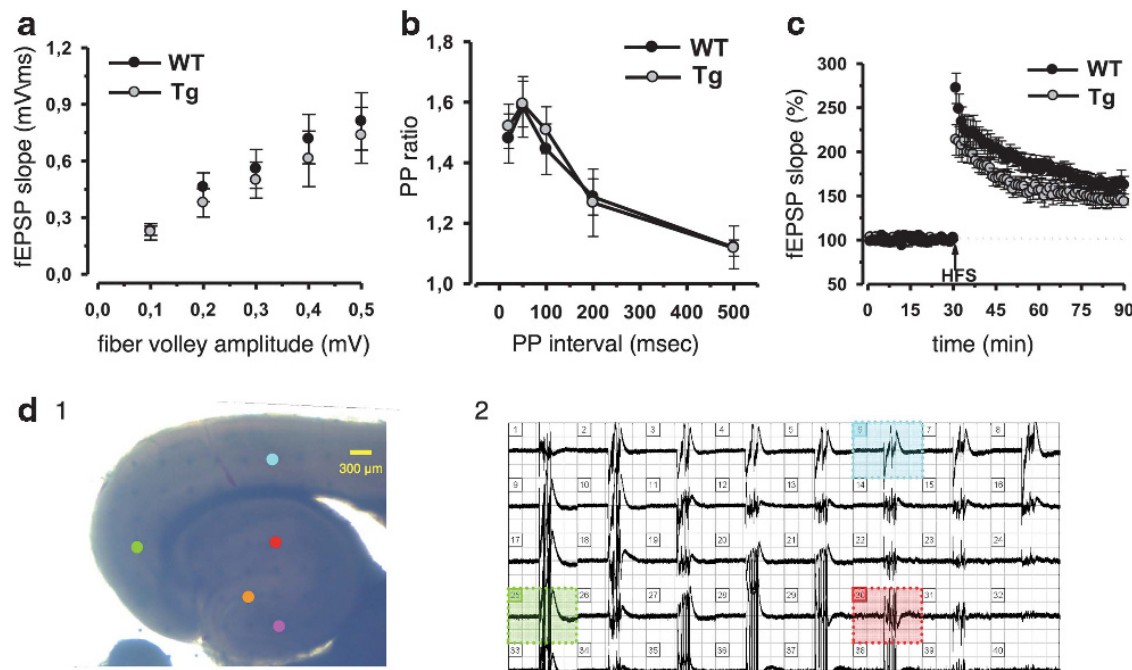

3
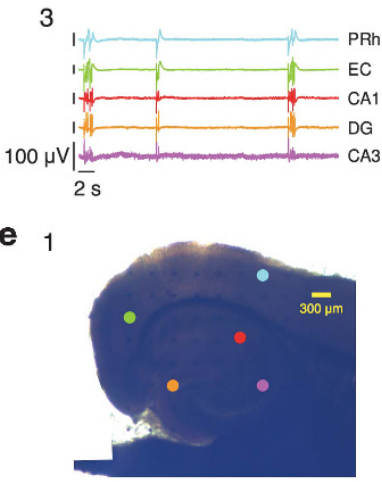

3

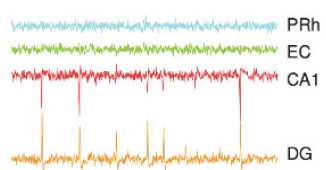

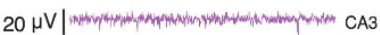
$2 \mathrm{~s}$

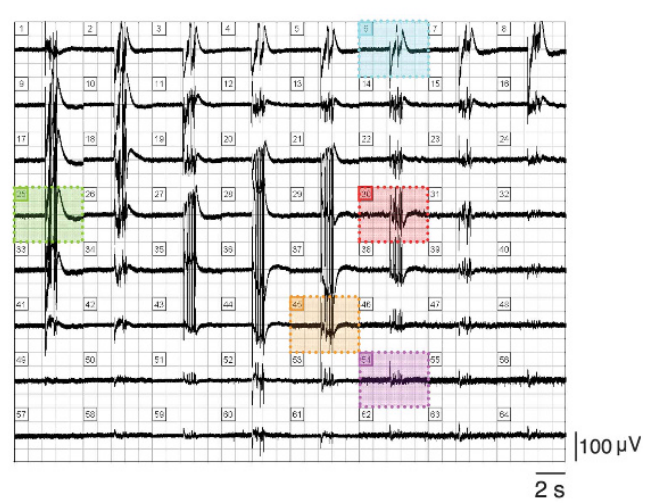

2

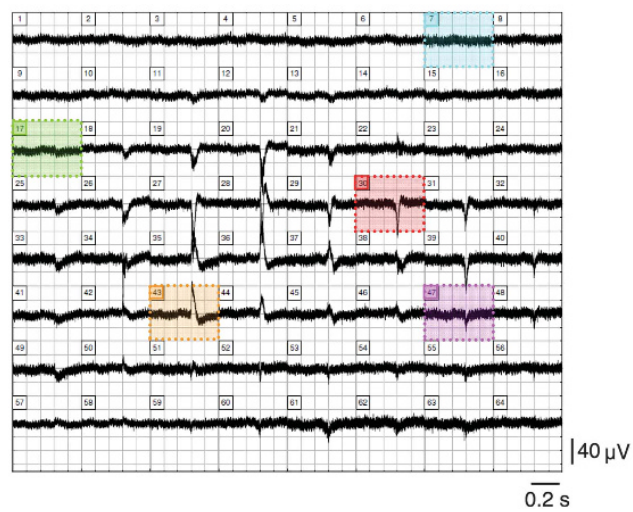

Figure 7 Electrophysiological analysis on the hippocampus of TgproNGF mice. Synaptic function in area CA1 and spontaneous epileptic-like events in the EC-hippocampal area of 12-month-old Tg proNGF\#3 and TG proNGF\#72 mice. (a) Input-output relation of fEPSPs as a function of presynaptic fiber volley size was similar for both TgproNGF\#3 $(n=8)$ and control mice $(n=9)$. (b) Comparison of paired-pulse facilitation (PPF) in TgproNGF\#3 $(n=7)$ and control slices $(n=8)$. Data are presented as the mean ( \pm S.E.M.) facilitation of the second response relative to the first response. (c) Superimposed pooled data showing the normalized changes in field potential slope ( \pm S.E.M.) in control $(n=9)$ and TgproNGF\#3 $(n=7)$ mice induced by high-frequency stimulation (HFS) protocol. (d and e) Multi-electrode array recordings from an EC-hippocampal slice of a TgproNGF\#3 (d1) and a TgproNGF\#72 (e1) mouse, placed over 64 planar electrodes, detectable in transparency through the slice. Colored dots indicate position of the electrodes, whose voltage signals appear in the respective panels 2 and 3 . (d2 and e2) Simultaneous recordings from each of the 64 electrodes. Colored areas highlight voltage traces detected by the corresponding electrodes in (d1 and e1) respectively. (d3 and e3) Continuous recordings from the perirhinal cortex (PRh), entorhinal cortex (EC), CA1, dentate gyrus (DG) and CA3 hippocampal area. Each trace is color coded according to the position of its corresponding electrode in (d1 and e1) respectively. No repetitive epileptic-like events were observed in EC-hippocampal slices from WT mice (15 slices from 4 animals). (d) Repetitive spontaneous interictal-like events were detected in 8 out of 18 EC-hippocampal slices from TgproNGF\#3 mice $(n=4)$. (e) In TgproNGF\#72 mice, repetitive spontaneous interictal-like events were found in each one of the slices recorded ( $n=15$ from 3 animals)

including FAD-derived models, based on the amyloid mechanism, and models based on neurotrophic deficits, is an imbalance in excitatory and inhibitory pathways ${ }^{29-31}$ and a normal CA1 LTP. ${ }^{29,32}$ For this reason, the presence of spontaneous epileptic-like seizures was investigated in the limbic area of TgproNGF mice, with field recordings in combined entorhinal cortex (EC)-hippocampal slices, using a 64-channels multi-electrode array device. ${ }^{33,34}$ Although no repetitive epileptic-like events were observed in EC-hippocampal slices from WT mice (15 slices from 4 animals), repetitive spontaneous interictal-like events were detected in 8 out of 18 EC-hippocampal slices from TgproNGF\#3 mice ( $n=4$; Figure.7d). These events occurred synchronously either in the whole rhinal CTX and HP ( $n=4$ slices) or confined to rhinal CTX ( $n=2$ slices) and HP only ( $n=2$ slices). 
Age-matched TgproNGF\#72 mice were tested for the presence of spontaneous epileptic-like discharges in EC-hippocampal slices. Similar to what was observed in TgproNGF\#3 mice, repetitive spontaneous interictal-like events were found in each of the slices recorded $(n=15$ from 3 animals), being confined to the sole hippocampal area, with no clear recruitment of nearby entorhinal networks (Figure 7e).

ProNGF and NGF levels in TgproNGF mice. TgproNGF\#3 and TgproNGF\#72 mice share various phenotypic end points (behavioral deficits, cholinergic neurons or fiber boutons reduction, increase in $\mathrm{A} \beta$ peptide immunoreactivity), but the overall severity of the phenotype is much stronger in TgproNGF\#3 than in TgproNGF\#72 mice. We therefore investigated whether these differences could be ascribed to different transgene expression levels or to different relative levels of proNGF and NGF.

The relative expression of proNGF-mRNA in TgproNGF\#3 and TgproNGF\#72 mice, versus WT mice, was determined by real-time-PCR on RNA from different brain areas (CTX, HP and $B F$ ), with primers specific for endogenous and transgenic proNGF mRNA (Figure 8a). As expected, a much higher amount of total proNGF mRNA (including both endogenous and transgenic proNGF mRNA using $\mathrm{P} 1$ primer) was detected in both TgproNGF lines as compared with WT mice (Figure 8a, green bars). The levels of endogenous proNGF transcript (identified with primer P2B) in TgproNGF mice are similar to those in WT mice (Figure 8a, blue bars). Therefore, the large increment in total proNGF transcript levels in Tg mice is wholly attributable to the transgene (directly measured with primer P2A encompassing the mutagenized consensus site for furin Figure $8 a$ and Supplementary Table S3A and B). Although a higher transgene copy number is often more prone to negative regulation of expression, ${ }^{23}$ this was not the case in TgproNGF\#72 mice. Surprisingly, the level of total proNGF mRNA is higher in the transgenic line TgproNGF\#72, showing a milder phenotype, than in TgproNGF\#3.

This apparent paradox prompted us to evaluate the levels of proNGF and mature NGF protein (Figure 1e). Anti-NGF immunoprecipitation (IP) with anti-NGF $\alpha$ D11 mAb, ${ }^{35}$ followed by western blot (challenged with anti-NGF or anti-proNGF

a
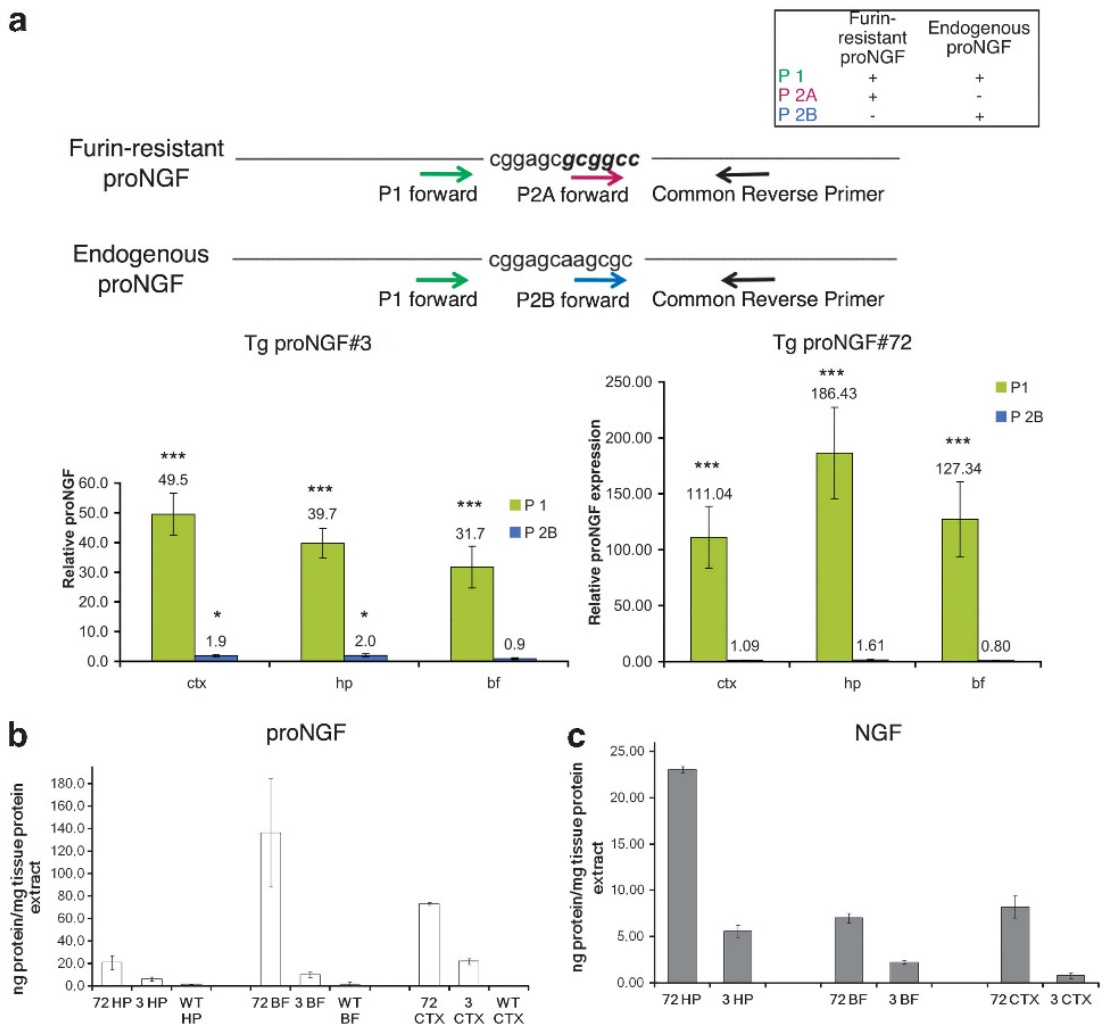

Figure 8 Expression of proNGF transgene (mRNA and protein). (a) ProNGF mRNA expression in brain areas (cortex (CTX), hippocampus (HP) and basal forebrain (BF)) of TgproNGF\#72 and \#3 mice by real-time quantitative RT-PCR. Schematic representation of the recognition sites of PCR primers used for quantitative real-time RT-PCR on endogenous and mutagenized proNGF nucleotide sequences. The specificity of real-time primers for endogenous proNGF mRNA and $\backslash$ or mutagenized proNGF mRNA encoded by the transgene is shown. Fold increase in proNGF expression (relative proNGF expression ratio) in TgproNGF\#3 versus WT mice (left panel). High amount of proNGF mRNA is detected in 3-month-old TgproNGF\#3 mice. Fold increase in proNGF expression (relative proNGF expression ratio in TgproNGF\#72 versus WT mice) (right panel). High amount of proNGF mRNA is detected in P90 TgproNGF\#72 mice. Bars and lines are representative of mean \pm S.E.M. (b and $\mathbf{c}$ ) Analysis of proNGF and mature NGF in specific brain areas (CTX, HP and BF) of TgproNGF\#72 and \#3 mice by immunoprecipitation and western blot. Immunoprecipitation on extracts from various brain areas (HP, BF and CTX) was performed with anti-NGF $\alpha$ D11 antibody, followed by western blot (WB) with anti-NGF or anti-proNGF antibody. After anti-NGF immunoprecipitation, the proNGF bands, in western blot probed with anti-proNGF, and the NGF bands, in the western blot probed with anti-NGF antibody, were quantified. The resulting intensities were normalized against the area of the bands, and then compared with an internal standard of recombinant proNGF and NGF. Loaded samples were in the linear range of detection. Comparison between proNGF (b) and NGF (c) amounts in TgproNGF\#72, \#3 and WT are reported in the histograms 
primary antibodies, Figure 1e), on extracts from various brain areas (CTX, HP and BF) from TgproNGF and WT mice revealed that proNGF content in both transgenic lines was much higher than in WT. Also, the levels of mature NGF were high in both transgenic lines, whereas in WT mice the band corresponding to mature NGF was undetectable (Figure 1e), as previously reported. ${ }^{3,36}$ ProNGF and NGF bands were identified by MALDI-TOF mass spectrometry (Supplementary Figure S5A, Supplementary Table S4). The densitometric analysis of immunoprecipitated proNGF and NGF showed that homozygous TgproNGF\#72 mice presented higher levels of not only proNGF but also NGF with respect to TgproNGF\#3 (Figure 8B and C), although the proNGF $\backslash$ NGF ratio was comparable in the two lines (Supplementary Figure S5B). Thus, in TgproNGF\#72 mice a higher NGF cortical and hippocampal content is associated with a milder phenotype, even in the presence of a high proNGF level. Conversely, in TgproNGF\#3 mice, lower levels of mature NGF are unable to compensate for the neurodegeneration-inducing effects of proNGF. ProNGF and NGF levels were also determined at P30, before the onset of cholinergic deficit, showing that both proNGF and NGF start to accumulate (Supplementary Figure S6) in both Tg lines; when IP enrichment is omitted (not shown), only proNGF is visualized. Noticeably, whereas proNGF and NGF levels increase over time, from P30 to P90 in different brain areas, in the CTX, NGF level decreases over time by a 0.6 factor, concomitantly to the increase of proNGF and the onset of cholinergic deficit.

\section{Discussion}

Growing evidence relates an increase in proNGF to aging and neurodegenerative diseases, such as $A D .^{3,19}$

In the AD11 anti-NGF mice, ${ }^{28}$ selective neutralization of mature NGF versus proNGF by the transgenic antibodies ${ }^{35}$ leads to a progressive Alzheimer's-like neurodegeneration, suggesting that imbalance in proNGF/NGF levels is an upstream driver of neurodegeneration. ${ }^{17}$ Consistently, crossing $A D 11$ to $p 75^{N T R}$ gene knockout mice rescues cholinergic deficit and decreases $\mathrm{A} \beta$ accumulation. ${ }^{18}$

However, a direct proof for a causal link between increased proNGF signaling and $\mathrm{A} \beta$-related neurodegeneration is lacking. In order to fill this gap and to gain a deeper insight into the functions of proNGF in the brain, we generated transgenic mice expressing furin-resistant proNGF. The study was focused on the line showing the strongest phenotype, TgproNGF\#3. Salient aspects of its phenotype were confirmed, in milder forms, in the independent line TgproNGF\#72. Of the other founders, the transgenic line TgproNGF\#36 showed reduced avoidance responses, but a severely impaired fertility prevented further analysis. TgproNGF\#17 mice presented hydrocephalus, and therefore were not analyzed further; interestingly, congenital hydrocephalus in children is associated with increased NGF-immunoreactive proteins (thus, NGF or proNGF) in the liquor. ${ }^{37}$

TgproNGF\#3 mice displayed a neurodegenerative phenotype characterized by severe learning and memory impairments, cholinergic deficit, increased APP processing into the $\mathrm{A} \beta$ amyloidogenic pathway and electrophysiological evidence of spontaneous epileptic-like events. At variance with the acute intracerebroventricular proNGF injections in rodents, ${ }^{38,39}$ chronic exposure to high proNGF levels determines signs of $A D$ neurodegeneration in transgenic mice. Although no abnormal tau pathology was found, a significant positivity for $\mathrm{A} \beta$ and oligomeric $\mathrm{A} \beta$ species was detected. $\mathrm{A}$ marked increase in diffuse $A \beta$ immunoreactivity was found throughout the CTX and HP of TgproNGF\#3 mice, either because of increased APP processing or impaired $\mathrm{A} \beta$ peptide clearance. A specific antibody probe ${ }^{26}$ for the oligomeric state of $A \beta(1-42)$, the most synaptotoxic forms of $A \beta$ peptide $(\mathrm{A} \beta \mathrm{O}),{ }^{25,40}$ revealed specific $\mathrm{A} \beta \mathrm{O}$ immunoreactivity in the $\mathrm{EC}$ (layer II) and in hippocampal pyramidal neurons, especially in the $\mathrm{CA} 1$ region. Of note, $\mathrm{APP} / \mathrm{A} \beta$ dysmetabolism in $\mathrm{EC}$ neurons is the starting cause of transsynaptic deficits in the cortical-hippocampal network in mouse models. ${ }^{41}$

Neurodegeneration in TgproNGF\#3 mice is characterized by astrocytic atrophy in 3-month-old mice, and by astrocytosis and marked alteration of astrocyte morphology in aged mice. In neurodegeneration models, early stages are often associated with atrophy of astroglia, whereas later, astrocytosis and astrocyte activation contribute to the neuroinflammatory component of neurodegeneration. ${ }^{42,43}$ Noteworthy, it was shown that proNGF produced by reactive astrocytes in response to oxidative stress promotes motoneuron death in a $75^{\mathrm{NTR}}$-dependent manner. ${ }^{44}$

Global multi-electrode array analysis in EC-hippocampal slices demonstrated spontaneous epileptic-like events in the CTX and HP of TgproNGF\#3 mice and HP of TgproNGF\#72 mice. Interestingly, both Down syndrome (DS) and AD are characterized by increased seizure activity ${ }^{29,30,45-47}$ and abnormal excitatory $\backslash$ inhibitory(E/I) balance. ${ }^{48}$ Of note, in the adult HP of anti-NGF AD11 mice, a shift of GABAergic signaling to the depolarizing direction is observed. ${ }^{31}$ Proneurotrophin secretion was reported in BF astrocytes after kainic acid-induced seizures. ${ }^{49}$ We therefore envisage a negative cycle whereby elevated proNGF alters the $\mathrm{E} / \mathrm{l}$ balance in the HP that, in turn, further contributes to elevate secretion of proNGF by astrocytes.

A shift toward amyloidogenic metabolism of APP can affect NGF metabolism, decreasing proNGF processing and inducing NGF degradation, switching the balance in favor of the precursor. ${ }^{6,7}$ Here, we provide evidence for the reciprocal arm of a vicious circle, demonstrating that experimentally increasing proNGF triggers cholinergic deficit and amyloidrelated neurodegeneration. ProNGF and amyloid metabolism might thus interplay in bidirectional way as part of a circular loop: the increase in proNGF induces an amyloidogenic metabolism of APP, with $A \beta$ oligomer formation, and vice versa (Supplementary Figure S7). Elevation of proNGF levels and $E / I$ imbalance, leading to spontaneous epileptic-like events, also form a negative feedback cycle that can be part of the same vicious cycle, leading to neurodegeneration (Supplementary Figure S7).

We conclude that high levels of proNGF determine a comprehensive neurodegenerative phenotype.

Some AD mouse models accumulate proNGF, suggesting that accumulation is an effect of the disease; ${ }^{50}$ this paper shows that accumulation of proNGF itself might cause downstream behavioral dysfunction, which parallels the degree of cholinergic deficit. Interestingly, mild cognitive 
impairment (MCI) and $A D$ patients show alteration in proNGFmediated pathways during $A D$ progression, ${ }^{51}$ and loss of NGF $\backslash$ trkA signaling in conditional KO mice is not sufficient to induce cognitive impairment. ${ }^{52}$ This underlines the importance of proNGF to NGF imbalance for the progression of neurodegeneration. ${ }^{17,18}$ Our results provide valuable information on the consequences of increasing proNGF levels, as observed in pathological conditions, ${ }^{21,51}$ even if the levels of proNGF observed in the derived transgenic mice are higher that the twofold increase of proNGF observed in AD brains. ${ }^{3}$ It is noteworthy that in the TgproNGF\#72 line, showing a milder phenotype, the level of mature NGF in the CTX is higher compared with TgproNGF\#3 mice. It will be interesting to evaluate whether perisomatic glia is involved in promoting a trophic outcome, mediated by NGF and trkA, in TgproNGF\#72 mice (by means of increased matrix metalloproteinase (MMP) production and extracellular cleavage of proNGF, as described $^{53}$ ). We did not observe increased hippocampal p75 ${ }^{\text {NTR }}$ levels, as recently described in AD. ${ }^{54}$

The phenotype observed in TgproNGF\#3 mice is tied to a specific transgene expression effect, as no cholinegic deficit is observed at P15 and P30, when both proNGF and NGF start to accumulate (Supplementary Figure S6). Noticeably, the level of NGF in the CTX decreases over time in both Tg lines, concomitantly to the increase of proNGF and the onset of cholinergic and behavioral phenotype. Thus, a relatively high proNGF level for a short time is not sufficient to determine the cholinergic and behavioral phenotype, at least at P30. Rather, its persistence over time, and the relative concurrent levels of mature NGF, appear to be relevant for the onset of the observed phenotype.

Our findings are significant in light of reports indicating elevated levels of proNGF in the brains of AD patients, ${ }^{3,19}$ and further validate the therapeutic concept of increasing levels of mature NGF as a strategy to treat AD. ${ }^{55}$

\section{Materials and Methods \\ Plasmid construction and generation of transgenic mice. The mouse pre-proNGF cDNA (obtained as described in Paoletti et al. ${ }^{56}$ ) was mutagenized at the furin cleavage site $^{8}$ and placed under the trans- criptional control of the mouse Thy1.2 promoter, driving brain-specific expression in postnatal and adult transgenic mice. ${ }^{22,57}$ The transcriptional unit also contains the EGFP gene, cloned downstream the furin-resistant pre-proNGF (see Supplementary Methods). After isolation of the DNA fragment encompassing the whole transcriptional unit, transgenic mice were generated by pronuclear DNA injection of zygotes C57B//6xDBA/2 F2 generation using standard procedures $^{58}$ (see Supplementary Methods for details).}

Animal handling and experiments. All experiments with mice were performed according to the national and international laws for laboratory animal welfare and experimentation (EEC council directive 86/609, 12 December 1987). Mice were kept under a 12-h dark to light cycle, with food and water ad libitum.

Behavioral analysis. Mice were kept under a 12-h dark to light cycle, with food and water ad libitum. Experiments were performed according to the national and international laws for laboratory animal welfare and experimentation (EEC council directive 86/609, 12 December 1987).

Active avoidance: The same apparatus was used to measure active avoidance and spontaneous locomotor activity. ${ }^{59}$ The apparatus was computer controlled and consisted of eight shuttle boxes, each divided into two $20 \times 10 \mathrm{~cm}$ compartments, connected by a $3 \times 3 \mathrm{~cm}$ opening. In avoidance training, a light $(10 \mathrm{~W})$ was switched on alternately in the two compartments and used as a conditioned stimulus (CS). The CS preceded the onset of the unconditioned stimulus (US) by $5 \mathrm{~s}$ and overlapped it for $25 \mathrm{~s}$. The US was an electric shock $(0.2 \mathrm{~mA})$ applied continuously to the grid floor. The intertrial interval was $30 \mathrm{~s}$. An avoidance response was recorded when the animal avoided the US by running into the dark compartment within $5 \mathrm{~s}$ after the onset of the CS. If animals failed to avoid the shock, they could escape it by crossing during the US. Mice were subjected to four daily 100-trial avoidance sessions.

Morris water maze: To establish whether proNGF mice show deficits in spatial memory, the Morris water maze test was used. A circular water tank made from aluminum (diameter: $120 \mathrm{~cm}$ ) was filled to a depth of $30 \mathrm{~cm}$ with water $\left(23 \pm 2^{\circ} \mathrm{C}\right)$ and rendered opaque by the addition of a small amount of white children finger paint. Four positions around the edge of the tank were arbitrarily designated north $(N)$, south $(S)$, east $(E)$ and west $(W)$, which provided four alternative start positions and also defined the division of the tank into four quadrants: NE, SE, SW and NW. A circular clear plastic escape platform (diameter $10 \mathrm{~cm}$ ) was submerged $0.5 \mathrm{~cm}$ below the water surface and placed at the midpoint of one of the four quadrants. Mice were trained for four trials per day (with an intertrial interval of $30 \mathrm{~min}$ ). The start position (N, S, E or W) was pseudorandomized across trials. The hidden platform remained in the NE quadrant. Mice were allowed up to $60 \mathrm{~s}$ to locate the escape platform, and their escape latency was recorded. At the end of the first trial of the first day, if the mice did not find the platform, they were placed for $20 \mathrm{~s}$ on the platform.

On the fifth day, the mice received a single probe trial, during which the escape platform was removed from the tank, and the swimming path of each mouse was video recorded over $60 \mathrm{~s}$ while it searched for the missing platform. The time spent in each quadrant was calculated.

Visual object recognition test (VORT): The apparatus consisted of a square arena $(60 \times 60 \times 30 \mathrm{~cm})$ constructed in PVC with black walls and white floor. The objects to be discriminated were made of plastic and glass and were too heavy to be displaced by the mice. The box and objects were cleaned up between trials to stop the build-up of olfactory cues.

During the first day (habituation phase), mice received two sessions of 5-min duration in the empty box to help them habituate to the apparatus and test room. The second session was delayed $30 \mathrm{~min}$ with respect to the end of the first one.

The next day, each mouse was placed in the box and exposed to two identical sample objects (objects A1 and A2) for $10 \mathrm{~min}$. This trial was called 'sample phase'. The experimenter measured the total time spent exploring each of the two objects. After a 24-h retention interval, the experimenter removed both objects and replaced one of the two by its identical copy (A3; so to ensure that there was no carryover of olfactory cues) and the other object (object B). Then, the mice were placed back in the box and exposed to the familiar object $A 3$ and to a novel test object $B$ for further $7 \mathrm{~min}$. The objects were placed in the same locations as the previous ones. The experimenter measured again the total time spent exploring each of the two objects ('test period'). All trials were recorded using a video camera connected to Any-maze software version 4.5 (Stoelting, Wood Dale, IL, USA).

Statistical analysis for behavioral data. All values of behavioral tests are expressed as mean \pm S.E.M. of 6-12 animals per group. AA data were evaluated by a two-factor analysis of variance (ANOVA) for repeated measures, the factor being the transgenic line (between-subject factor) and daily sessions (within-subject factor). Locomotor activity data were analyzed using one-way ANOVA. The post hoc comparisons were carried out using Fisher's test. MWM (at all ages), immobility and the sample phase of the ORT at 3 months of age were analyzed using one-way ANOVA. The post hoc comparisons were carried out using the Bonferroni test. The comparison between new and familiar object in the ORT at all ages and the sample phase of the ORT at 12 months of age were analyzed by paired $t$-test. The statistical significance was set at $P<0.05$ in all the analyses.

Histological analysis. After behavioral analysis, proNGF and WT mice were anesthetized with an excess of 2,2,2-tribromethanol $(400 \mathrm{mg} / \mathrm{kg})$ and intracardially perfused with a saline solution following a $4 \%$ solution of paraformaldehyde in phosphate-buffered saline (PBS). Brains were postfixed for $24 \mathrm{~h}$ and then transferred in $30 \%$ sucrose/PBS solution and sectioned on a sliding freezing microtome (Leica, Wetzlar, Germany). Next, $40 \mu \mathrm{m}$ coronal sections were collected in $0.05 \%$ sodium azide/PBS in $1.5 \mathrm{ml}$ tubes and stored at $4^{\circ} \mathrm{C}$ until usage. Immunoperoxidase and or immunofluorescence were performed as described in Supplementary Methods. 
Electrophysiology. WT and TgproNGF\#3 mice (12 to 14 months old) were anesthetized by inhalation of 2-Bromo-2-Chloro-1,1,1-trifluoroethane and killed by decapitation. Experiments were carried out in accordance with the Declaration of Helsinki Principles and followed international guidelines on the ethical use of animals from the European Communities Council Directive of 24 November 1986 $(86 / 609 / E E C)$. All experimental procedures are described in detail in Supplementary Method and in references 33 and 60.

RNA isolation and real-time qRT-PCR. RNA was isolated, quality controlled and RNA was subjected to quantitative real-time RT-PCR (qRT-PCR) as described in Supplementary Methods. Primers for qRT-PCR are described in Supplementary Methods; Section 5: RNA isolation and Real-Time qRT-PCR.

Immunoprecipitation and western blot. Different brain areas (CTX, HP and BF) were isolated from WT and heterozygous TgproNGF\#3 male mice. For each experiment, a pool of four animals was used and analyzed. Each pool of cerebral areas was homogenized with $2 \mathrm{ml}$ of Lysis buffer per $\mathrm{g}$ of tissue (Lysis buffer: Tris $\mathrm{HCl} 0.1 \mathrm{M}, \mathrm{NaCl} 0.4 \mathrm{M}$, SDS $0.1 \%$, Triton X-100 1\%). The homogenates were incubated in ice for $30 \mathrm{~min}$ and then centrifuged for $30-40 \mathrm{~min}$ at $15000 \times \mathrm{g}$ at $4^{\circ} \mathrm{C}$. The total amount of protein in the supernatant was quantified by Bradford Assay (SIGMA-Aldrich, St Louis, MO, USA).

The same amount of total protein samples $(4 \mathrm{mg}$ ) was immunoprecipitated with a large excess of anti-NGF $\alpha$ D11 antibody before western blot. The whole samples after IP were loaded onto the gel (criterion, 4-12\% BIS-Tris, $12+2$ ) for western blotting. The primary antibody used was anti-proNGF (Alomone, Jerusalem, Israel) and the secondary antibody was goat anti-rabbit HRP conjugated (Jackson, West Grove, PA, USA). Recombinant proNGF and NGF were used as control; loaded samples were in the linear range of detection.

The membrane was then stripped and incubated with the primary antibody, anti-NGF M20 (Santa Cruz, Heidelberg, Germany).

The intensity of the bands on the western blots from three separate experiments was measured by densitometric analysis using the software of the Kodak scanner. The net intensity was normalized against the internal area of each band and then compared with an internal standard of NGF or proNGF.

\section{Conflict of Interest}

The authors declare no conflict of interest.

Acknowledgements. This paper is dedicated to our beloved friend and coauthor Cecilia Tiveron, who made a crucial contribution to this project before she passed away on 17 December 2010, as a tribute to her enthusiasm and dedication to science and to her friendship. We also dedicate the paper to Professor Rita Levi-Montalcini, Nobel Laureate founder of the EBRI institute, tireless and enthusiastic example for all of us. This work was funded by EU FP6 MEMORIES Project (no. 037831), Telethon grant no. GGP05234, the Italian Institute of Technology (IIT), the Italian Ministry of Higher Education and Scientific Research (FIRB no. RBAP10L8TY) and Fondazione Roma. We thank Domenico Vignone for valuable help with the immunohistochemistry experiments and Andrea Urbani for advise on the mass spectrometry analysis.

1. Francke $U$, de Martinville $B$, Coussens $L, U$ Ulrich $A$. The human gene for the beta subunit of nerve growth factor is located on the proximal short arm of chromosome. Science 1983; 222: $1248-1251$.

2. Scott J, Selby M, Urdea M, Quiroga M, Bell Gl, Rutter WJ. Isolation and nucleotide sequence of a cDNA encoding the precursor of mouse nerve growth factor. Nature 1983; 302: $538-540$.

3. Fahnestock M, Michalski B, Xu B, Coughlin MD. The precursor pro-nerve growth factor is the predominant form of nerve growth factor in brain and is increased in Alzheimer's disease. Mol Cell Neurosci 2001; 18: 210-220.

4. Lee R, Kermani P, Teng KK, Hempstead BL. Regulation of cell survival by secreted proneurotrophins. Science 2001; 294: 1945-1948.

5. Seidah NG, Benjannet S, Pareek S, Savaria D, Hamelin J, Goulet B et al. Cellular processing of the nerve growth factor precursor by the mammalian pro-protein convertases. Biochem J 1996; 314(Pt 3): 951-960.

6. Bruno MA, Cuello AC. Activity-dependent release of precursor nerve growth factor, conversion to mature nerve growth factor, and its degradation by a protease cascade. Proc Natl Acad Sci USA 2006; 103: 6735-6740.
7. Bruno MA, Leon WC, Fragoso G, Mushynski WE, Almazan G, Cuello AC. Amyloid betainduced nerve growth factor dysmetabolism in Alzheimer disease. J Neuropathol Exp Neurol 2009; 68: 857-869

8. Beattie MS, Harrington AW, Lee R, Kim JY, Boyce SL, Longo FM et al. ProNGF induces p75-mediated death of oligodendrocytes following spinal cord injury. Neuron 2002; 36: 375-386.

9. Harrington AW, Leiner B, Blechschmitt C, Arevalo JC, Lee R, Mörl K et al. Secreted proNGF is a pathophysiological death-inducing ligand after adult CNS injury. Proc Natl Acad Sci USA 2004; 101: 6226-6230.

10. Nykjaer A, Lee R, Teng KK, Jansen $P$, Madsen $P$, Nielsen MS et al. Sortilin is essential for proNGF-induced neuronal cell death. Nature 2004; 427: 843-848.

11. D'Onofrio M, Paoletti F, Arisi I, Brandi R, Malerba F, Fasulo $L$ et al. NGF and proNGF regulate functionally distinct mRNAs in $\mathrm{PC} 12$ cells: an early gene expression profiling. PLoS One 2011; 6: e20839.

12. Rattenholl A, Lilie H, Grossmann A, Stern A, Schwarz E, Rudolph R. The pro-sequence facilitates folding of human nerve growth factor from Escherichia coli inclusion bodies. Eur $J$ Biochem 2001; 268: 3296-3303.

13. Kaplan DR, Martin-Zanca D, Parada LF. Tyrosine phosphorylation and tyrosine kinase activity of the trk proto-oncogene product induced by NGF. Nature 1991; 350 158-160

14. Klein R, Jing SQ, Nanduri V, O'Rourke E, Barbacid M. The trk proto-oncogene encodes a receptor for nerve growth factor. Cell 1991; 65: 189-197.

15. Puglielli L. Aging of the brain, neurotrophin signaling, and Alzheimer's disease: is IGF1-R the common culprit? Neurobiol Aging 2008; 29: 795-811.

16. Masoudi R, loannou MS, Coughlin MD, Pagadala P, Neet KE, Clewes $O$ et al. Biological activity of nerve growth factor precursor is dependent upon relative levels of its receptors. J Biol Chem 2009; 284: 18424-18433.

17. Capsoni S, Cattaneo A. On the molecular basis linking nerve growth factor (NGF) to Alzheimer's disease. Cell Mol Neurobiol 2006; 26: 619-633.

18. Capsoni S, Tiveron C, Vignone D, Amato $G$, Cattaneo A. Dissecting the involvement of tropomyosin-related kinase $A$ and p75 neurotrophin receptor signaling in NGF deficitinduced neurodegeneration. Proc Natl Acad Sci USA 2010; 107: 12299-12304.

19. Peng S, Wuu J, Mufson EJ, Fahnestock M. Increased proNGF levels in subjects with mild cognitive impairment and mild Alzheimer disease. J Neuropathol Exp Neurol 2004; 63: 641-649.

20. Ginsberg SD, Che S, Wuu J, Counts SE, Mufson EJ. Down regulation of trk but not p75NTR gene expression in single cholinergic basal forebrain neurons mark the progression of Alzheimer's disease. J Neurochem 2006; 97: 475-487.

21. Counts SE, Mufson EJ. The role of nerve growth factor receptors in cholinergic basal forebrain degeneration in prodromal alzheimer disease. J Neuropathol Exp Neurol 2005; 64: 263-272.

22. Caroni P. Overexpression of growth-associated proteins in the neurons of adult transgenic mice. J. Neurosci Methods 1997; 71: 3-9.

23. Feng G, Mellor RH, Bernstein M, Keller-Peck C, Nguyen QT, Wallace M et al. Imaging neuronal subsets in transgenic mice expressing multiple spectral variants of GFP. Neuron 2000; 28: 41-51.

24. Li Z, Burrone J, Tyler WJ, Hartman KN, Albeanu DF, Murthy VN. Synaptic vesicle recycling studied in transgenic mice expressing synaptopHluorin. Proc Natl Acad Sci USA 2005; 102 : 6131-6136.

25. Haass C, Selkoe DJ. Soluble protein oligomers in neurodegeneration: lessons from the Alzheimer's amyloid beta-peptide. Nat Rev Mol Cell Biol 2007; 8: 101-112.

26. Meli G, Visintin M, Cannistraci I, Cattaneo A. Direct in vivo intracellular selection of conformation-sensitive antibody domains targeting Alzheimer's amyloid-beta oligomers. $\mathrm{J} \mathrm{Mol} \mathrm{Biol} \mathrm{2009;} \mathrm{387:} \mathrm{584-606.}$

27. Holcomb L, Gordon MN, McGowan E, Yu X, Benkovic S, Jantzen P et al. Accelerated Alzheimer-type phenotype in transgenic mice carrying both mutant amyloid precursor protein and presenilin 1 transgenes. Nat Med 1998; 4: 97-100.

28. Capsoni S, Ugolini G, Comparini A, Ruberti F, Berardi N, Cattaneo A. Alzheimer-like neurodegeneration in aged antinerve growth factor transgenic mice. Proc Natl Acad Sci USA 2000; 97: 6826-6831.

29. Palop JJ, Chin J, Roberson ED, Wang J, Thwin MT, Bien-Ly N et al. Aberrant excitatory neuronal activity and compensatory remodeling of inhibitory hippocampal circuits in mouse models of Alzheimer's disease. Neuron 2007; 55: 697-711.

30. Palop JJ, Mucke L. Epilepsy and cognitive impairments in Alzheimer disease. Arch Neurol 2009; 66: 435-440.

31. Lagostena L, Rosato-Siri M, D'Onofrio M, Brandi R, Arisi I, Capsoni S et al. In the adult hippocampus, chronic nerve growth factor deprivation shifts GABAergic signaling from the hyperpolarizing to the depolarizing direction. J Neurosci 2010; 30: 885-893.

32. Rosato-Siri M, Cattaneo A, Cherubini E. Nicotine-induced enhancement of synaptic plasticity at CA3-CA1 synapses requires GABAergic interneurons in adult anti-NGF mice. J Physiol 2006; 576(Pt 2): 361-377.

33. Berretta N, Ledonne A, Mango D, Bernardi G, Mercuri NB. Hippocampus vs. entorhinal cortex decoupling by an NR2 subunit-specific block of NMDA receptors in a rat in vitro model of temporal lobe epilepsy. Epilepsia 2012; 53: e80-e84.

34. Avoli M, D'Antuono M, Louvel J, Köhling R, Biagini G, Pumain R et al. Network and pharmacological mechanisms leading to epileptiform synchronization in the limbic system in vitro. Prog Neurobiol 2002; 68: 167-207. 
35. Cattaneo A, Rapposelli B, Calissano P. Three distinct types of monoclonal antibodies after long-term immunization of rats with mouse nerve growth factor. J Neurochem 1988; 50: 1003-1010.

36. Reinshagen M, Geerling I, Eysselein VE, Adler G, Huff KR, Moore GP et al. Commercial recombinant human beta-nerve growth factor and adult rat dorsal root ganglia contain an identical molecular species of nerve growth factor prohormone. J Neurochem 2000; 74: 2127-2133.

37. Salehi Z, Mashayekhi F. Nerve growth factor level in the cerebrospinal fluid in hydrocephalus. Cerebrospinal Fluid Research 2004; 1(Suppl 1): S34.

38. Fortress AM, Buhusi M, Helke KL, Granholm AC, Cholinergic AC. Degeneration and alterations in the TrkA and p75NTR balance as a result of pro-NGF injection into aged rats. J Aging Res 2011; 2011: 460543.

39. Kichev A, llieva EV, Piñol-Ripoll G, Podlesniy P, Ferrer I, Portero-Otín M et al. Cell death and learning impairment in mice caused by in vitro modified pro-NGF can be related to its increased oxidative modifications in Alzheimer disease. Am J Pathol 2009; 175: 2574-2585.

40. Shankar GM, Walsh DM. Alzheimer's disease: synaptic dysfunction and A-beta. Mol Neurodegener 2009; 23: 48.

41. Harris JA, Devidze N, Verret L, Ho K, Halabisky B, Thwin MT et al. Transsynaptic progression of amyloid- $\beta$-induced neuronal dysfunction within the entorhinal-hippocampal network. Neuron 2010; 68: 428-441.

42. Verkhratsky A, Olabarria M, Noristani HN, Yeh CY, Rodriguez JJ. Astrocytes in Alzheimer's disease. Neurotherapeutics 2010; 7: 399-412.

43. Li C, Zhao R, Gao K, Wei Z, Yin MY, Lau LT et al. Astrocytes: implications for neuroinflammatory pathogenesis of Alzheimer's disease. Curr Alzheimer Res 2011; 18:67-80.

44. Domeniconi M, Hempstead BL, Chao MV. Pro-NGF secreted by astrocytes promotes motor neuron cell death. Mol Cell Neurosci 2007; 34: 271-279.

45. Menéndez M. Down syndrome, Alzheimer's disease and seizures. Brain Dev 2005; 27: 246-252.

46. Abou-Khalil BW. How important is Alzheimer's disease as a risk factor for unprovoked seizures and epilepsy in the elderly? Epilepsy Curr 2010; 10: 36-37.

47. De Simone R, Puig XS, Gélisse P, Crespel A, Genton P. Senile myoclonic epilepsy: delineation of a common condition associated with Alzheimer's disease in Down syndrome. Seizure 2010; 19: 383-389.
48. Rissman RA, Mobley WC. Implications for treatment: GABAA receptors in aging, Down syndrome and Alzheimer's disease. J Neurochem 2011; 117: 613-622.

49. Volosin M, Song W, Almeida RD, Kaplan DR, Hempstead BL, Friedman WJ. Interaction of survival and death signaling in basal forebrain neurons: roles of neurotrophins and proneurotrophins. J Neurosci 2006; 26: 7756-7766.

50. Perez SE, He B, Muhammad N, Oh KJ, Fahnestock M, Ikonomovic MD et al. Cholinotrophic basal forebrain system alterations in 3xTg-AD transgenic mice. Neurobiol Dis 2011; 41: 338-352.

51. Mufson EJ, He B, Nadeem M, Perez SE, Counts SE, Leurgans S et al. Hippocampal ProNGF signaling pathways and $\beta$-amyloid levels in mild cognitive impairment and Alzheimer disease. J Neuropathol Exp Neurol 2012; 71: 1018-1029.

52. Müller M, Triaca V, Besusso D, Costanzi M, Horn JM, Koudelka J et al. Loss of NGF-TrkA signaling from the CNS is not sufficient to induce cognitive impairments in young adult or intermediate-aged mice. J Neurosci 2012; 32: 14885-14898.

53. Kalous A, Nangle MR, Anastasia A, Hempstead BL, Keast JR. Neurotrophic actions initiated by proNGF in adult sensory neurons may require peri-somatic glia to drive local cleavage to NGF. J Neurochem 2012; 122: 523-536.

54. Chakravarthy B, Ménard M, Ito S, Gaudet C, Dal Prà I, Armato U et al. Hippocampal membrane-associated p75NTR levels are increased in Alzheimer's disease. J Alzheimer Dis 2012; 30: 675-684.

55. Cattaneo A, Capsoni S, Paoletti F. Towards non invasive nerve growth factor therapies for Alzheimer's disease. J Alzheimers Dis 2008; 15: 255-283.

56. Paoletti F, Covaceuszach S, Konarev PV, Gonfloni S, Malerba F, Schwarz E et al. Intrinsic structural disorder of mouse proNGF. Proteins 2009; 75: 990-1009.

57. Vidal M, Morris R, Grosveld F, Spanopoulou E. Tissue specific control elements of the thygene. EMBO J 1990; 9: 833-840.

58. Ciana P, Braliou GG, Demay FG, Von Lindern M, Barettino D, Beug $\mathrm{H}$ et al. Leukemic transformation by the v-ErbA oncoprotein entails constitutive binding to and repression of an erythroid enhancer in vivo. EMBO J 1998; 17: 7382-7394.

59. Pavone F, Battaglia M, Sansone M. Prevention of amitriptyline-induced avoidance impairment by tacrine in mice. Behav Brain Res 1997; 89: 229-236.

60. Middei S, Roberto A, Berretta N, Panico MB, Lista S, Bernardi G et al. Learning discloses abnormal structural and functional plasticity at hippocampal synapses in the APP23 mouse model of Alzheimer's disease). Learn Mem 2010; 17: 236-240. 